\title{
End to end simulator for the WIVERN W-band Doppler conically scanning spaceborne radar
}

\author{
Alessandro Battaglia ${ }^{1,2,3}$, Paolo Martire ${ }^{1}$, Eric Caubet $^{4}$, Laurent Phalippou ${ }^{4}$, Fabrizio Stesina ${ }^{1}$, Pavlos \\ Kollias $^{5,6}$, and Anthony Illingworth ${ }^{7}$ \\ ${ }^{1}$ Politecnico of Torino, Italy \\ ${ }^{2}$ University of Leicester, UK \\ ${ }^{3}$ National Centre for Earth Observation, UK \\ ${ }^{4}$ Thales Alenia Space, Toulouse, France \\ ${ }^{5}$ Department of Environmental and Climate Sciences, Brookhaven National Laboratory, Upton, NY, USA \\ ${ }^{6}$ Division of Atmospheric Sciences, Stony Brook University, Stony Brook, NY, USA \\ ${ }^{7}$ University of Reading, Reading, UK
}

Correspondence: Alessandro Battaglia

alessandro_battaglia@polito.it

\begin{abstract}
The WIVERN (WInd VElocity Radar Nephoscope) mission, soon entering in Phase-0 of the ESA Earth Explorer program, promises to complement Doppler wind lidar by globally observing, for the first time, vertical profiles of winds in cloudy areas. This work describes an end to end simulator of the WIVERN conically scanning $94 \mathrm{GHz}$ Doppler radar, the only payload of the mission. Specific features of the simulator are: the conically scanning geometry; the inclusion of cross-polarization effects and of the simulation of a radiometric mode; the applicability to global cloud model outputs via an orbital model; the incorporation of a mispointing model accounting for thermo-elastic distortions, microvibrations, startrackers uncertainties, etc.; the inclusion of the surface clutter. Some of the simulator capabilities are showcased for a case study involving a full rotational scan of the instrument.

The simulator represents a very useful tool for studying the performances of the WIVERN concept and possible trade-offs for the different configurations (e.g. different antenna sizes, pulse lengths, antenna patterns, ...). Thanks to its modular structure the simulator can be easily adapted to different orbits, different scanning geometries and different frequencies.
\end{abstract}

\section{Introduction}

Accurate forecasts save lives, support emergency management and the mitigation of impacts, thus preventing losses from severe weather while creating substantial revenue (Bauer et al., 2015). Windstorms are the largest contributor to economic losses caused by weather related hazards, resulting in approximately 500 billion USD (adjusted to 2011) of global damage over the last decade. Together with floods they are the costliest natural hazards in Europe: they account for more than 30\% (60\%) of total (insured) losses (https://ec.europa.eu/jrc/sites/jrcsh/files/pesetaiv_task_13_windstorms_final_report.pdf). The Aeolus wind lidar have demonstrated a large impact in reducing forecast errors when assimilated by European Weather Forecasting Centers 
(Rennie et al., 2021). In addition to winds, cloud and precipitation measurements remain key for both Numerical Weather Prediction (NWP) applications and for advancing understanding of cloud processes and their role in climate simulations.

The WIVERN (WInd VElocity Radar Nephoscope) concept has been recently proposed within the ESA Earth Explorer 11 call in order to strengthen the wind, cloud and precipitation observation capability of the Global Observing System. The mission has been selected for Phase 0 studies. It hinges upon a single instrument: a Dual-Polarization Doppler W-band scanning cloud radar with a 3-m circular aperture non-deployable main reflector. The WIVERN antenna conically scans around nadir at an off-nadir angle of $38^{\circ}$ at 12 Revolution Per Minute (RPM). This rotation speed implies the use of one horn for transmission and another one for reception. Flying on a 500-km orbit, the instrument provides a swath of $800 \mathrm{~km}$ (see Fig. 1).

The aim of the mission is to complement Doppler lidar winds acquired in clear sky conditions and from the tops of optically thick clouds (Rennie et al., 2021) and other wind observations (at cloud top via geostationary observation derived atmospheric motion vector, close to the ocean surface via scatterometers, via radio soundings) by observations in areas of optically thick clouds, critical for cyclogenesis, that cannot be seen by optical sensors. Observations in these areas have the largest potential to improve forecasts (McNally, 2002). Therefore the WIVERN mission is expected to provide:

- unprecedented wind observations inside tropical cyclones and mid-latitude windstorms that will routinely reveal the dynamic structure of such destructive systems;

- observations of convective motions that will validate the representation of convection in models;

- global profiles of cloud properties and precipitation over an $800 \mathrm{~km}$ swath that will better quantify the hydrological cycle and the atmospheric and surface energy budget;

- first direct observation of tropospheric winds that will underpin the predictions of transport and dispersion of trace gases and pollutants in atmospheric chemistry and air quality models.

These advances in the observational capabilities are expected to address three science objectives, with immediate applications and societal benefits.

1. to extend the lead time of useful prediction skills of hazardous weather (e.g., wind-storm, cyclones, floods) by direct assimilation of wide-swath winds from clouds and profiles of radar reflectivity of clouds and precipitation into numerical weather prediction (NWP) models.

2. to improve numerical models by providing new metrics and observational verification to assess different NWP parameterisation schemes within such models. NWP and climate models use similar schemes so better NWP models will also augment confidence in climate models.

3. to establish a benchmark for the climate record of cloud profiles, global solid/light precipitation and, for the first time, in-cloud winds, crucial for a better quantification of the Earth's hydrological cycle, and energy budgets, with a significant reduction in sampling errors of current and planned cloud radar missions. 
Table 1. WMO (World Meteorological Organisation) requirements for horizontal winds for numerical weather prediction (NWP) and the expected performance of WIVERN.

\begin{tabular}{|c|c|c|c|c|}
\hline & Uncertainty & Horizontal Resolution & Vertical Resolution & Observing Cycle \\
\hline Goal & $2 \mathrm{~m} / \mathrm{s}$ & $15 \mathrm{~km}$ & $0.5 \mathrm{~km}$ & $1 \mathrm{~h}$ \\
\hline Breakthrough & $3 \mathrm{~m} / \mathrm{s}$ & $100 \mathrm{~km}$ & $1 \mathrm{~km}$ & $6 \mathrm{~h}$ \\
\hline Threshold & $5 \mathrm{~m} / \mathrm{s}$ & $100 \mathrm{~km}$ & $3 \mathrm{~km}$ & $12 \mathrm{~h}$ \\
\hline WIVERN & $2 \mathrm{~m} / \mathrm{s}$ & $20 \mathrm{~km}$ & $0.64 \mathrm{~km}$ & 1 day $^{\star}$ \\
\hline
\end{tabular}

${ }^{\star}$ Global average between $\pm 82^{\circ}$ latitude.

WMO requirements for data assimilation into global NWP (Illingworth et al., 2018a) can be found at OSCAR (https://www.wmosat.info/oscar/) and are summarised in Tab. 1. The threshold of $12 \mathrm{~h}$ for the observing cycle is quite demanding; three scatterometers with 1200-km swaths can approach this revisit time. Noticeably, the Aeolus non-scanning narrow swath clear sky wind measurements are having a significant effect despite their typical clear-sky uncertainty of 4-5 m/s and their coarse sampling (Rennie et al., 2021); thus, even winds with uncertainty above the WMO threshold and with sampling below threshold have proved extremely valuable for NWP. Horanyi et al. (2014) showed that assimilating winds biased by $1-2 \mathrm{~m} / \mathrm{s}$ when the random error is around $2 \mathrm{~m} / \mathrm{s}$ would degrade the forecast so a bias of less than $1 \mathrm{~m} / \mathrm{s}$ should be added to the specifications of Tab. 1.

In order to achieve these targets WIVERN will adopt:

1. polarization diversity to overcome both the range-Doppler dilemma and the short decorrelation times produced by the Doppler fading associated with the low Earth orbiting satellite velocity (Battaglia et al., 2013);

2. a large antenna $(3 \mathrm{~m})$ to achieve a narrow beam, thus a fine vertical resolution and fewer issues related to non uniform beam filling (NUBF) biases (Tanelli et al., 2002).

Previous studies (Illingworth et al., 2018b; Battaglia et al., 2018), based on the CloudSat climatology of cloud reflectivities, have demonstrated that the WIVERN radar should observe between one and two million winds per day that satisfy the WMO "goal" of $2 \mathrm{~m} / \mathrm{s}$ precision. However it is important to define a rigorous framework where to assess the accuracy and precision of Doppler velocities. For instance errors introduced by satellite mispointing induced by orbital-dependent thermo-elastic distortion of the antenna, by the solar array drive mechanism microvibrations, by the rotating antenna vibration, etc., can seriously affect space-borne Doppler measurements, as previously studied in Doppler scanning radars (Ardhuin et al., 2019) and in Doppler lidars (Weiler et al., 2021).

End to end (E2E) simulators are paramount tools for evaluating instrument performances in preparatory mission studies. Several radar simulators have been developed in the recent years to simulate space-borne radars [e.g. Haynes et al. (2007); Matsui et al. (2013); Dellaripa et al. (2021)]. Novelty of this work is that our radar simulator is tailored to conically scanning Doppler radars adopting polarization diversity, as envisaged for the WIVERN mission. The simulator also incorporates a 
model accounting for mispointing as potentially caused by different sources like thermo-elastic distortions, micro-vibrations, star-trackers uncertainties, it includes an orbital model and accounts for surface clutter. Sect. 2 provides a detailed description of all the modules of the E2E simulator whereas Sect. 3 presents some applications, with examples extracted from a case study and a first assessment of some of the errors related to the Doppler measurements. Conclusions and future work are discussed in Sect. 4.

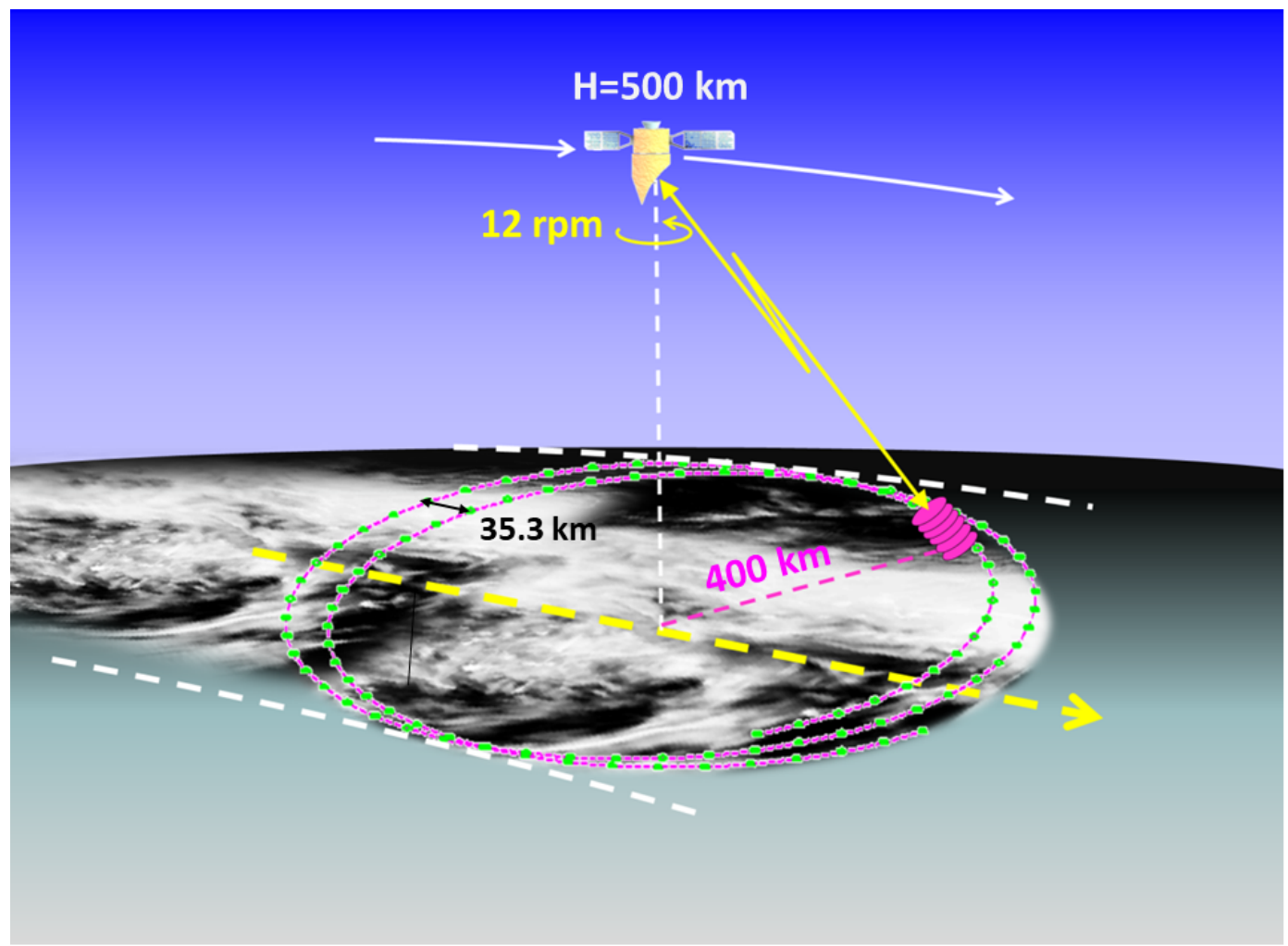

Figure 1. Artistic impression of the WIVERN concept: a 94-GHz Doppler radar with 3-m antenna scanning at 12 rpm tracing out a cycloidal track with an incidence angle of $41.6^{\circ}$.

\section{The E2E simulator}

Our simulator has been developed within the ESA Earth Explorer program and it exploits recent development of radar simulators. In particular it benefits from the inclusion of polarization diversity pulse pair processing and wide swath scanning (Battaglia et al., 2013; Battaglia and Kollias, 2015), the effect of the "ghosts" introduced by the cross-talk (Wolde et al., 10 2019) between the $\mathrm{H}$ and $\mathrm{V}$ channels caused by strongly reflective depolarising targets and surface clutter as derived from airborne measurements (Battaglia et al., 2017) and the simulation of passive mode to provide brightness temperatures at W-band 
(Battaglia and Panegrossi, 2020). A simplified 2D-version of the simulator has recently been applied to CloudSat observations and co-located ECMWF 3D winds to provide an intial assessment of errors introduced by different sources related to NUBF, aliasing, averaging and to the noise in the Doppler spectra estimators (Battaglia et al., 2018).

The WIVERN simulator can cope with data produced by state-of-the-art high-resolution cloud-resolving models as the 5 basis for creating scenes that are used as input to various radiative transfer programs and instrument simulation modules. This provides the ability to simulate satellite overpasses and subsequent measurement processes. In this control environment, forward and retrieval models can be evaluated and compared against the "truth" of the input model scene. Similarly each error source can be evaluated separately based on the assumption that, as a first approximation, the different error sources can be assumed independent, so that the total quadratic error (bias) can be computed as a quadratic sum (an absolute sum) of the different errors (Battaglia and Kollias, 2015). For instance, the satellite motion NUBF-induced errors can be estimated by computing the velocities running the simulator with or without satellite motion and then taking the differences of the two (Battaglia et al., 2018).

A schematic for the overall structure of the simulator is depicted in Fig. 2 with a list of current and potential additional capabilities tabulated in Tab. 2. A global model provides high resolution 3D scenes with clouds and winds; outputs of the global model are used as inputs of a forward model that computes ideal profiles of W-band co- and cross-polar reflectivities and Doppler velocities; the forward model outputs are then combined in a pulse-pair signal processing module which adds the proper noise levels to produce WIVERN outputs ( $\mathrm{H}$ and V-channel reflectivities and line of sight Doppler velocities). The description of the different modules is detailed in the following subsections. The radar specifics used throughout this paper are the ones recently proposed to the ESA Earth Explorer 11 and are listed in Tab. 3.

Table 2. Current and future capabilities of the WIVERN E2E simulator.

\begin{tabular}{|c|c|c|}
\hline Capability & Current & Future/Desirable \\
\hline Model input & Global $(4.3 \mathrm{~km}$ hor. res.) & Global $(<1 \mathrm{~km}$ hor. res. $)$ \\
\hline Antenna pattern & Gaussian & Any (abulated shape \\
\hline Point target response & Top hat & $K_{D P}, \rho_{H V}$ \\
\hline$\sigma_{0}$ modelling & Constant over ocean/land & Linked to surface properties (roughness, vegetation type, soil moisture, etc) \\
\hline Simulated radar variables & $Z_{c o}, v_{D}, L D R$ & Based on Hogan and Battaglia (2008) \\
\hline Multiple scattering flag & None & \\
\hline
\end{tabular}

\subsection{The System for Atmospheric Modeling (SAM) global storm resolving model}

The Global Storm-Resolving Models (GSRM, Stevens et al. (2019); Satoh et al. (2019)) are a new class of high resolution global numerical models that explicitly simulate small scales of motions coupled to large-scale circulation systems. This allows GSRMs to explicitly resolve deep convection and thus overcome challenges arising from deep convection parameterizations 


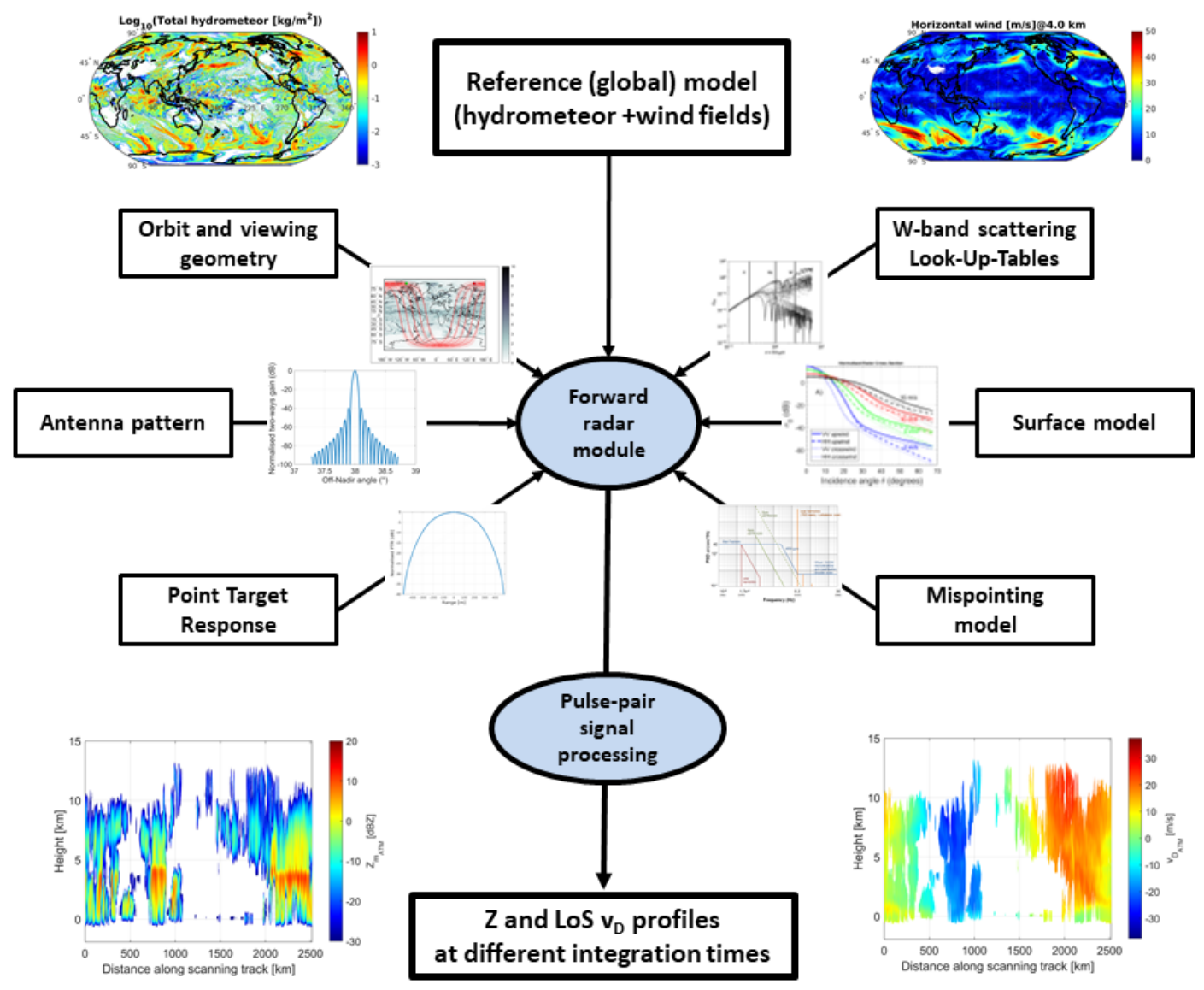

Figure 2. Flow chart illustrating the overall structure of the Wivern E2E simulator. The integrated hydrometeor content and the $4.0 \mathrm{~km}$ height winds are shown at the top as examples of input fields from the reference global model whereas outputs of the simulator [reflectivities and line-of-sight (LOS) winds] for a WIVERN "cross section" that will be examined later (Figs. 11-14) are presented in the bottom coloured panels.

(Kendon et al., 2017). The first intercomparison of GSRMs was conducted in the context of the DYAMOND (the DYnamics of the Atmospheric general circulation On Non-hydrostatic Domains) project (Stevens et al., 2019).

Here, output from the GSRM that participated in the DYAMOND project, the System for Atmospheric Modeling (SAM, Khairoutdinov and Randall (2003) which employs an anelastic form of the non-hydrostatic equations was used as input to the

5 WIVERN radar simulator. The SAM has a horizontal resolution of $4.3 \mathrm{~km}$ and 74 vertical layers. Details of the SAM model configuration can be found in Stevens et al. (2019). The model output is available at the DYAMOND project web site via https://www.esiwace.eu/services/dyamond 
Table 3. Specifics of the radar for the simulation. The configuration here adopted is the one proposed for WIVERN in a recent ESA Earth Explorer 11 call. The E2E simulator can study various trade-offs to optimise mission, system and instrument parameters.

\begin{tabular}{|c|c|}
\hline Satellite altitude, $h_{\text {sat }}$ & $500 \mathrm{~km}$ \\
\hline Satellite velocity, $v_{\text {sat }}$ & $7600 \mathrm{~ms}^{-1}$ \\
\hline off-nadir pointing angle & $38^{\circ}$ \\
\hline Incidence angle, $\theta_{i}$ & $41.6^{\circ}$ \\
\hline RF output frequency & $94.05 \mathrm{GHz}$ \\
\hline Pulse width & $3.3 \mu \mathrm{s}$ \\
\hline Antenna beamwidth, $\theta_{3 d B}$ & $0.071^{\circ}$ \\
\hline Circular antenna diameter & $3 \mathrm{~m}$ \\
\hline Rotation speed & $12 \mathrm{rpm}$ \\
\hline Footprint speed & $500 \mathrm{kms}$ \\
\hline Transmit polarization & $\mathrm{H} \mathrm{or} \mathrm{V}$ \\
\hline Cross-polar isolation & $<-25 \mathrm{~dB}$ \\
\hline Single pulse sensitivity & $-18 \mathrm{dBZ}$ \\
\hline H-V Pair Repetition Frequency & $4 \mathrm{kHz}$ \\
\hline Range sampling distance (rate) & $100 \mathrm{~m}(1.5 \mathrm{MHz})$ \\
\hline Number of H-V Pairs per 1 km integration length & 8 \\
\hline
\end{tabular}

${ }^{\dagger}$ A value of $-15 \mathrm{dBZ}$ maybe assumed to allow for a $3 \mathrm{~dB}$ margin.

\subsection{Forward radar module}

\subsubsection{Orbital model and scanning geometry}

The orbit selected for WIVERN is sun-synchronous with a mean inclination of $97.4 \mathrm{deg}$, a mean eccentricity of 0.001257 , a mean local time of the ascending node equal $6: 00$ and $15+1 / 5$ orbits per day which provides global coverage up to $\pm 82^{\circ}$ latitudes.An example of the simulation of five orbits is shown in Fig. 3. By running several orbits it is possible to compute for each location the mean and maximum (i.e. the worst case scenario) revisit time of the WIVERN radar footprint; the latter is plotted as a function of latitude and longitude in the left panel of Fig. 4. The maximum revisit time has a strong latitudinal behaviour with a minimum in the equatorial band (peaking at more than 5 days) and a secondary peak at $\sim 46^{\circ}$ (exceeding 3 days at some longitudes). The maximum (blue line) and mean (red line) revisit time averaged over all longitudes as a function of latitude are shown in the right panel of Fig. 4. While the maximum revisit time presents different local maxima, the mean revisit time is monotonically decreasing from the Equator to the Poles with a mean value of 1.5 days in the Tropical band and of less than 1 day above $50^{\circ}$ latitude, which leads to an average global revisit time of once a day between $\pm 82 \mathrm{deg}$ latitude.

The radar is sounding the atmosphere down to the ground with a range resolution of $500 \mathrm{~m}$. Fig. 5 and Fig. 6 illustrate the observing slant geometry; the actual vertical resolution will be the result of the slant range resolution, the antenna beamwidth 


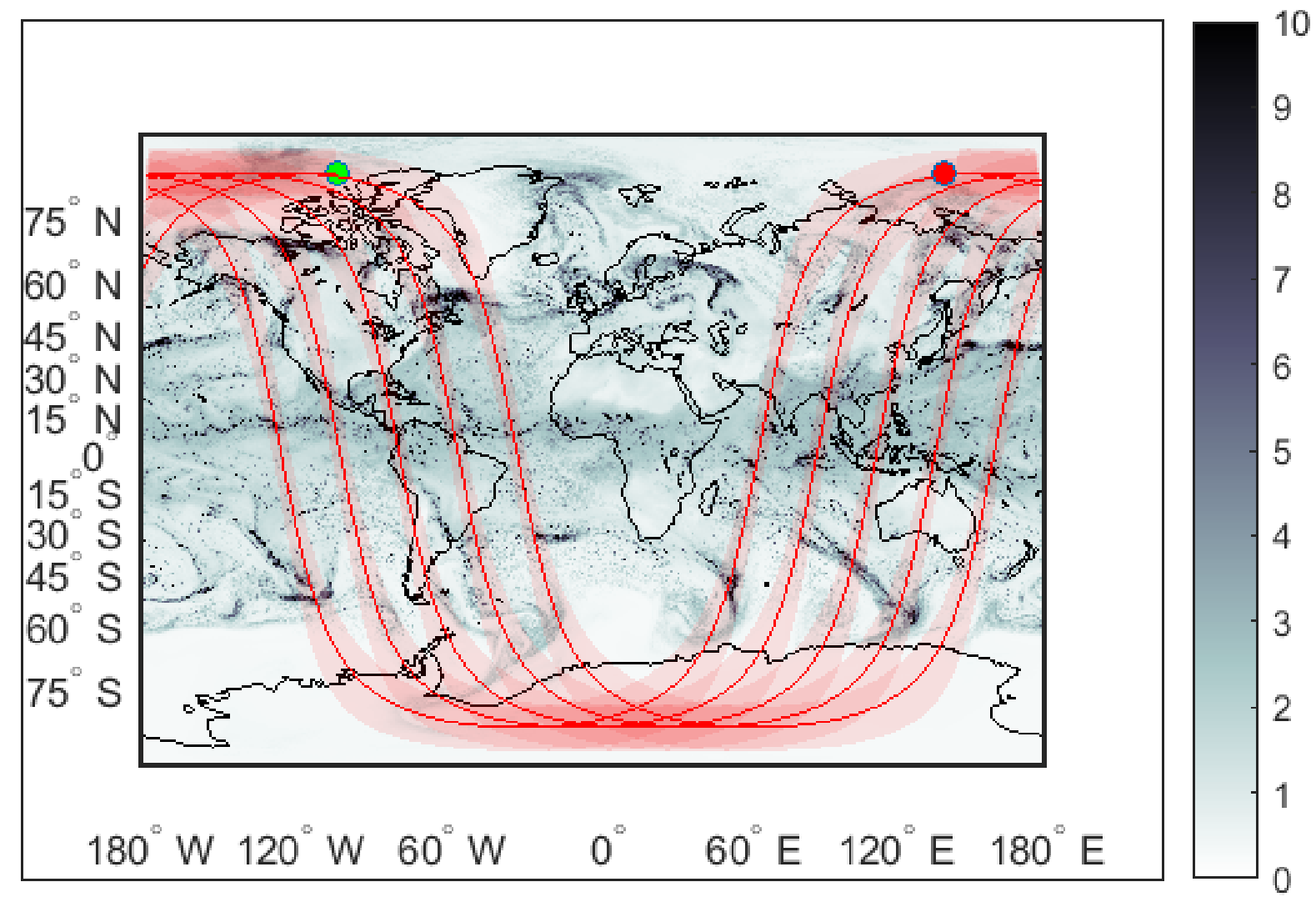

Figure 3. Example of simulation of five WIVERN orbits with the ground tracks (red lines), the $800 \mathrm{~km}$ WIVERN scanning swath (red-shaded region) plotted over the hydrometeor one-way path integrated $\mathrm{W}$-band attenuation (the colorbar scale is in $\mathrm{dB}$ ). A single model snapshot is used for the simulation.

and the satellite altitude (Meneghini and Kozu, 1990). Note that, for a uniform cloud, 90\% (99\%) of the backscattering power is coming from a region whose vertical extent is $640 \mathrm{~m}(980 \mathrm{~m})$. The horizontal sampling pattern is a function of the rotation speed. The values used here (Tab. 3) are the result of a preliminary optimization for wind product performance (sensitivity and spatial resolution).

\section{$5 \quad$ 2.2.2 W-band scattering Look Up Tables}

Scattering properties (extinction and backscattering coefficients, single scattering albedo and asymmetry parameters) at each model grid point are computed by adding up the contributions from the different hydrometeors (cloud water, cloud ice, rain, snow). The total scattering, backscattering and extinction coefficients are derived by adding up the single-particle scattering, backscattering and extinction cross sections for the different hydrometeor species according to their particle size distributions. The single scattering albedo is just the ratio between the scattering and the extinction coefficients; the asymmetry parameter 

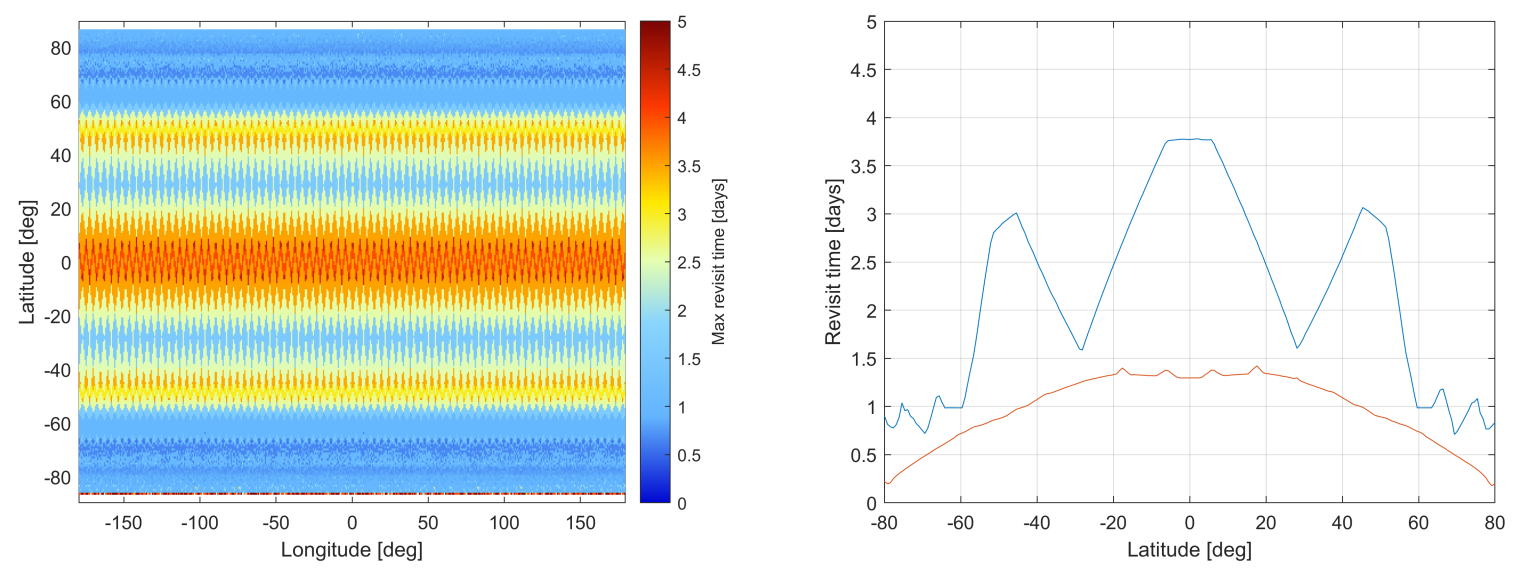

Figure 4. Left: WIVERN maximum (i.e. the worst case scenario) revisit time for a $15+1 / 5$ day orbit. Right: the red dotted and blue continuous lines correspond to the latitude-averaged mean and maximum revisit time, respectively, as a function of latitude. Note a mean revisit time of 1.5 days in the Tropical band and of less than 1 day above $50^{\circ}$ latitude. Globally, the mean revisit time is roughly daily.

Table 4. Range of values of reflectivities and extinction coefficients at W-band for different cloud types.

\begin{tabular}{|c|c|c|}
\hline Cloud type & Reflectivities & Extinction coefficients \\
\hline Cirrus & & negligible \\
Eccentricity & 0.001257 & 0.00135062 \\
Inclination [deg] & 97.418 & 97.4011 \\
Mean LTAN [hour] & 6.000 & -169.6234 \\
RAAN [deg] & -169.387 & 69.4689 \\
Argument of Perigee [deg] & 90.0 & -69.2253 \\
Mean anomaly @ $\mathrm{t}_{0}[\mathrm{deg}]$ & 0 & J2000 \\
\hline Epoch $\mathrm{t}_{0}$ & \multicolumn{2}{|c|}{$2019-01-0106: 00: 00$} \\
\hline Reference Frame & \multicolumn{2}{|c|}{ Jame } \\
\hline
\end{tabular}

is derived as a weighted average of the different species asymmetry parameters with the scattering coefficients as weights. Mie theory (Bohren and Huffman, 1983) is used to compute the single-particle scattering properties. The class "Snow" (which represents all large ice particles) is assumed to have a constant density of $0.1 \mathrm{~g} / \mathrm{cm}^{3}$. The simulator can accommodate ice species with different densities and axial ratio (e.g. a set of other Look-Up-Table from Rayleigh-Gans approximation is also available from previous studies (Mróz et al., 2021)); it only requires to switch the reference scattering Look-Up-Table.

In order to simulate the cross-polar reflectivities linear depolarization ratios $(L D R)$ values are assigned to the different hydrometeor species based on $L D R$ climatology collected at the Chilbolton observatory (see Battaglia et al. (2018)). The 


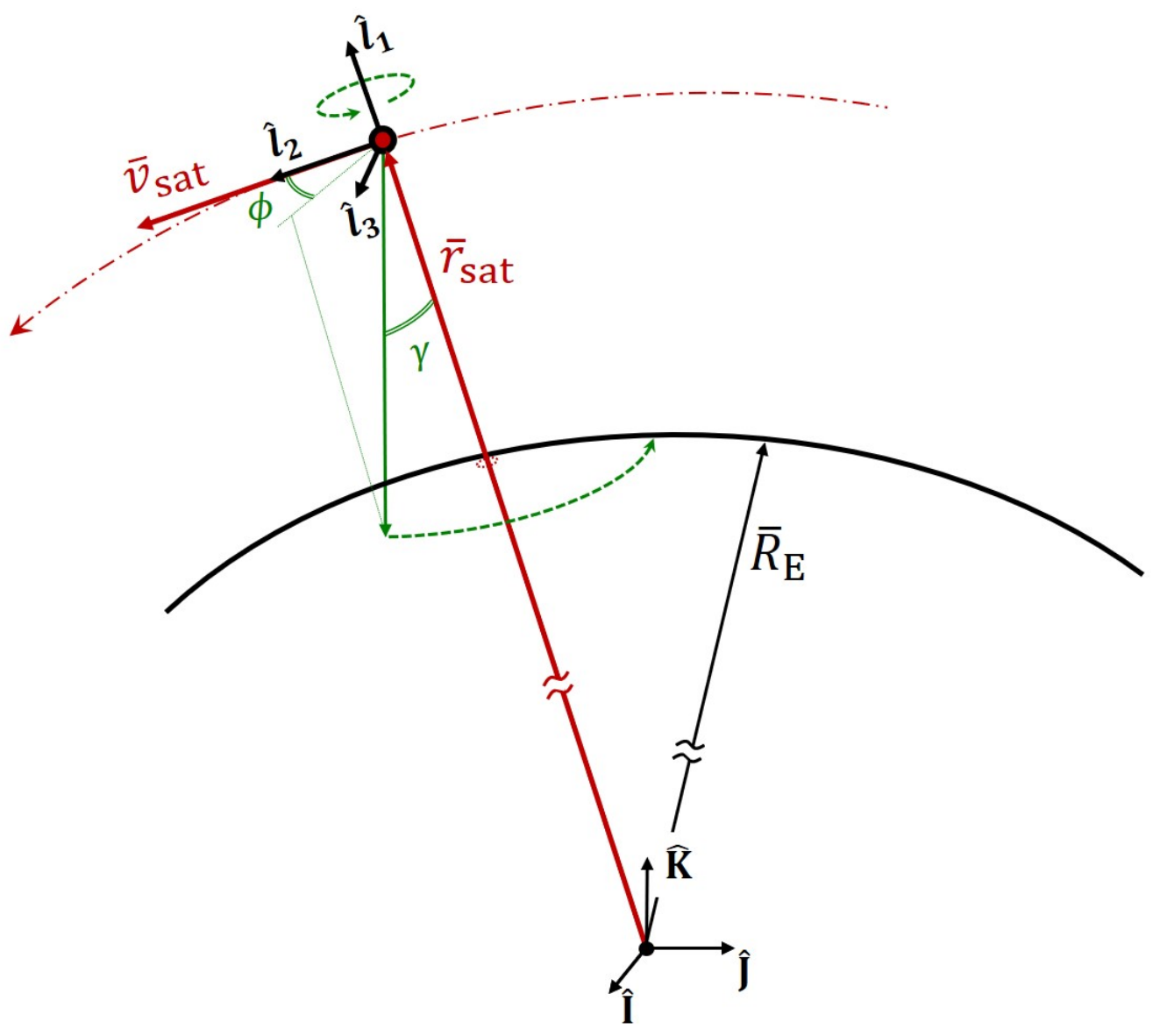

Figure 5. Illustration of the satellite scanning geometry. The boresight direction (solid green arrow) is identified by the elevation angle $\gamma=38^{\circ}$ with respect to the Nadir direction and by the azimuth angle $\phi$ measured from the horizontal direction $\hat{l}_{2}$ of the Local-Vertical/LocalHorizontal (LVLH) reference frame. The solid red arrows $\boldsymbol{r}_{\text {sat }}$ and $\boldsymbol{v}_{\text {sat }}$ represent the satellite's position and velocity vectors in the geocentricequatorial (IJK) reference frame.

different hydrometeors of the model ouput are assigned $L D R$ values drawn from a normal distribution with $1.5 \mathrm{~dB}$ standard deviation and mean values of $-21,-19,-19$ and $-30 \mathrm{~dB}$ for rain, ice crystals, snow and cloud, respectively. $L D R$ in the area of temperatures between the -1 and $+4^{\circ}$ isotherm (this, in a conservative approach, generally tends to overestimate the thickness of the melting layer) are assumed to have a mean value of $-14 \mathrm{~dB}$ and a standard deviation of $1.5 \mathrm{~dB}$. 


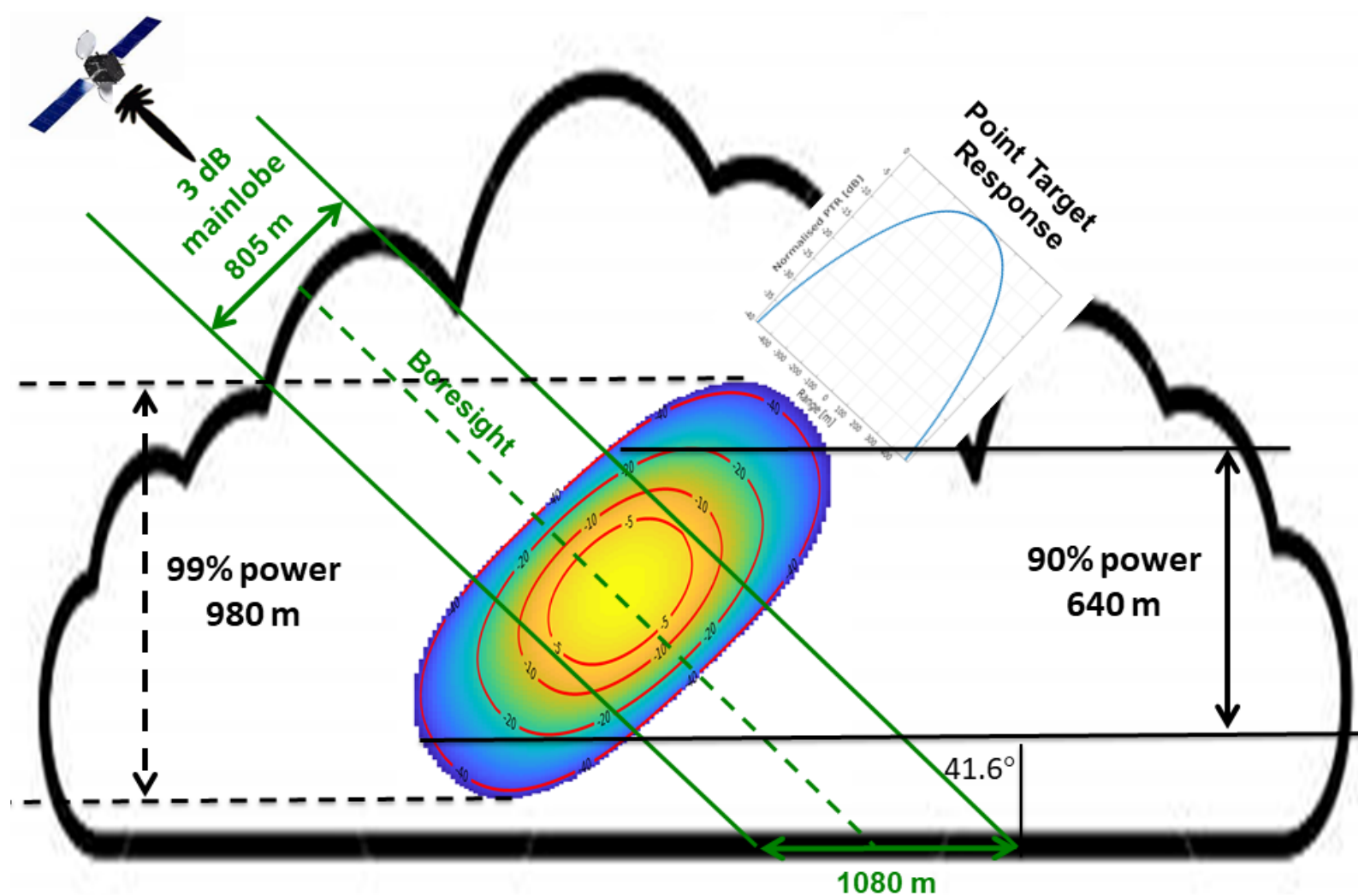

Figure 6. Schematic illustrating the WIVERN observing geometry. The specifics of the radar are detailed in Tab. 3

\subsubsection{Surface model}

Surface $\sigma_{0}$ are assumed to be normally distributed around $-25 \mathrm{~dB}$ and $-8 \mathrm{~dB}$ for sea and land respectively with $3 \mathrm{~dB}$ standard deviation whereas the surface $L D R$ is assumed to be $-14 \mathrm{~dB}$ and $-6 \mathrm{~dB}$ for sea and land with $1 \mathrm{~dB}$ standard deviation (Battaglia et al., 2017). In case of coastal regions a weighted mean accounting for the surface type fraction is taken.

\section{$5 \quad$ 2.2.4 Point target response}

The point target response (PTR) could be assumed to be a simple top hat with a pulse length, $\tau_{p}$, of $3.3 \mu$ s. More sophisticated PTR function could be used in order to optimise the equivalent noise bandwidth and PTR width. The PTR is used as convolution function along range for all the radar observables. 


\subsubsection{Antenna pattern}

Since the WIVERN antenna is circular a simple Gaussian antenna pattern is assumed with a one-way gain equal to:

$G\left(\theta_{a}\right)=G_{0} \exp \left[-4 \log (2)\left(\frac{\theta_{a}}{\theta_{3 d B}}\right)^{2}\right] \equiv G_{0} f_{a}\left(\theta_{a}\right)$,

where $G_{0}$ is the antenna gain in the boresight direction, $\theta_{a}$ is the antenna polar angle with respect to the boresight and $\theta_{3 d B}$

5 is the antenna 3-dB beamwidth. Any antenna pattern inclusive of side lobes can be added by simply sampling it on the angles used later on for the solid angle integration.

\subsection{Simulations of radar observables}

Both the volume scattering from the atmosphere and the surface scattering from the ground-return must be accounted for when computing the radar observables.

The power received by the radar from the atmosphere, $P_{r}^{a t m}(t)$ is given by an integral over the backscattering volume (Bringi and Chandrasekar, 2001):

$P_{r e c}^{a t m}(t)=P_{t r} \frac{G_{0}^{2} \lambda^{2}}{(4 \pi)^{3}} \int_{\phi_{a}=0}^{2 \pi} \int_{\theta_{a}=0}^{\pi} f_{a}^{2}\left(\theta_{a}\right) \int_{0}^{\infty} \frac{\eta\left(r, \theta_{a}, \phi_{a}\right)}{r^{2}}|P T R(t-2 r / c)|^{2} e^{-2 \int_{0}^{r} k_{e x t}(s) d s} d r d \Omega$

where $\eta$ is the radar reflectivity, $P_{t r}$ is the transmitted power, $\lambda$ is the wavelength of radar, $k_{e x t}$ is the extinction coefficient. Practically in order to compute the reflectivity factor corresponding to the atmosphere the three dimensional integral in Eq. (2) is first broken into an integral over the solid angle (defined with respect to the boresight direction); this allows computing $Z$ for ranges $r_{i}$ sampled at distance $\delta r(=100 \mathrm{~m}$ in our case but adjustable to the specific need):

$Z_{\delta r}^{a t m}(r)=\frac{\int_{\phi_{a}=0}^{2 \pi} \int_{\theta_{a}=0}^{\pi} f_{a}^{2}\left(\theta_{a}\right) Z_{e}\left(r, \theta_{a}, \phi_{a}\right) e^{-2 \int_{0}^{r} k_{e x t}(s) d s} d \Omega}{\int_{\phi_{a}=0}^{2 \pi} \int_{\theta_{a}=0}^{\pi} f_{a}^{2}\left(\theta_{a}\right) d \Omega} \equiv \frac{\int_{\phi_{a}=0}^{2 \pi} \int_{\theta_{a}=0}^{\pi} f_{a}^{2}\left(\theta_{a}\right) Z_{e}\left(r, \theta_{a}, \phi_{a}\right) e^{-2 \int_{0}^{r} k_{e x t}(s) d s} d \Omega}{\Omega_{2 A}}$

where $\Omega_{2 A}$ is the two-way antenna main-lobe solid angle (equal to $\pi \theta_{3 d B}^{2} /(8 \ln 2)$ for a Gaussian antenna); the solid angle integral is performed by sampling 7 polar and 21 azimuthal angles with respect to the antenna boresight. Then $Z_{\delta r}^{a t m}(r)$ is convoluted with the point target response:

$Z^{a t m}(r)=w_{P T R} * Z_{\delta r}^{a t m}(r)$

where $w_{P T R}$ is the normalised point target response.

The power received by the radar from the surface at a range $r, P_{r}^{\text {surf }}(r)$ is computed by an integration performed over the surface, $\Sigma$, which is obtained from the intersection between the surface and the spherical shell with radius between $r-\Delta r / 2$ and $r+\Delta r / 2$ with $\Delta r=c \tau_{p} / 2$ (Meneghini and Kozu, 1990):

$P_{\text {rec }}^{\text {surf }}(r)=P_{t r} \frac{G_{0}^{2} \lambda^{2}}{(4 \pi)^{3}} \int_{\Sigma} \frac{\sigma_{0}\left(\theta_{a}, \phi_{a}\right) f_{a}^{2}\left(\theta_{a}\right) e^{-2 \int_{0}^{r} k_{e x t}(s) d s}}{r^{4}} d \Sigma \equiv P_{t r} \frac{G_{0}^{2} \lambda^{2}}{(4 \pi)^{3}} \mathcal{I}_{\text {surf }}(r)$ 
where $\sigma_{0}$ is the normalised radar cross section (NRCS). The surface contribution can be written as an equivalent reflectivity term as:

$Z^{\text {surf }}(r)=\frac{\lambda^{4}}{\pi^{5}|K|^{2}} \frac{1}{\Omega_{2 A}} \frac{r^{2}}{\Delta r} \mathcal{I}_{\text {surf }}(r)$

The integral $\mathcal{I}_{\text {surf }}$ defined in Eq. (5) is evaluated by numerical integration on a $3 \mathrm{~km}^{2}$ grid defined on the plane tangent to the

5 Earth at the intersection between the Earth and the antenna boresight. Eqs. (5-6) have been applied to a smaller $\delta r(=100 \mathrm{~m})$ to compute $Z_{\delta r}^{\text {surf }}(r)$, similarly to what has been done in Eq. (2); then $Z^{\text {surf }}(r)$ can be computed with a formula analogous to Eq. (4).

The total reflectivity signal is obtained by adding up the atmospheric and the surface contributions, e.g. for the V-channel:

$Z_{V V}(r)=Z_{V V}^{\text {surf }}(r)+Z_{V V}^{a t m}(r)$

both are saved in order to compute the impact of the clutter on the radar observables at low altitudes.

To simulate a Doppler radar with polarization diversity profiles cross-polar returns are also needed. These are obtained performing the same integrals but using the cross-polar reflectivities via $L D R$ and the cross polar surface NRCS, $\sigma_{0}^{H V}$. The cross-polar reflectivities will be important to compute the appearance of the "ghosts" (Battaglia et al., 2013; Illingworth et al., 2018b; Wolde et al., 2019). WIVERN will transmit pairs of $3.3 \mu$ s long pulses at a pulse repetition frequency of $4 \mathrm{kHz}$, as illustrated in Fig. 7. The pulses will be sent in pairs alternatively horizontally and vertically polarised with a separation of $20 \mu \mathrm{s}$. This will achieve a maximum unambiguous velocity of $\pm 40 \mathrm{~m} / \mathrm{s}$, sufficiently high for unfolding the highest winds. The reflectivity signal received in the $\mathrm{V}$-channel, $Z_{V}$, is the combination of the co-polar V-signal, $Z_{V V}$ (continuous red line) combined with the anticipated cross talk of the $\mathrm{H}$-signal, $Z_{H V}$ (dashed red line):

$Z_{V}(r)=Z_{V V}(r)+Z_{H V}\left(r+c T_{h v} / 2\right)$

The hatched regions highlight the ranges where the cross-signal exceed the copolar signal and therefore will significantly modify the reflectivity signal.

Similarly the signal received in the H-channel, $Z_{H}$, is the combination of the co-polar H-signal, $Z_{H H}$, (continuous blue line) combined with the delayed cross talk of the V-signal, $Z_{V H}$, (not shown):

$Z_{H}(r)=Z_{H H}(r)+Z_{V H}\left(r-c T_{h v} / 2\right)$

The order of the polarization state of each pulse pairs is switched from pulse to pulse (see bottom panel in Fig. 7) in order to cancel out differential phase shift during propagation between the radar and the targets and for any difference in the lengths of the two polarization transmission lines (Pazmany et al., 1999). Therefore, if we assume no differential reflectivity $\left(Z_{H H}=\right.$ $\left.Z_{V V}=Z_{c o}\right)$, reciprocity $\left(Z_{H V}=Z_{V H}=Z_{c x}\right)$ and the same gain in the two linearly polarized channels, for $M$-pairs of pulses what is practically measured is : 


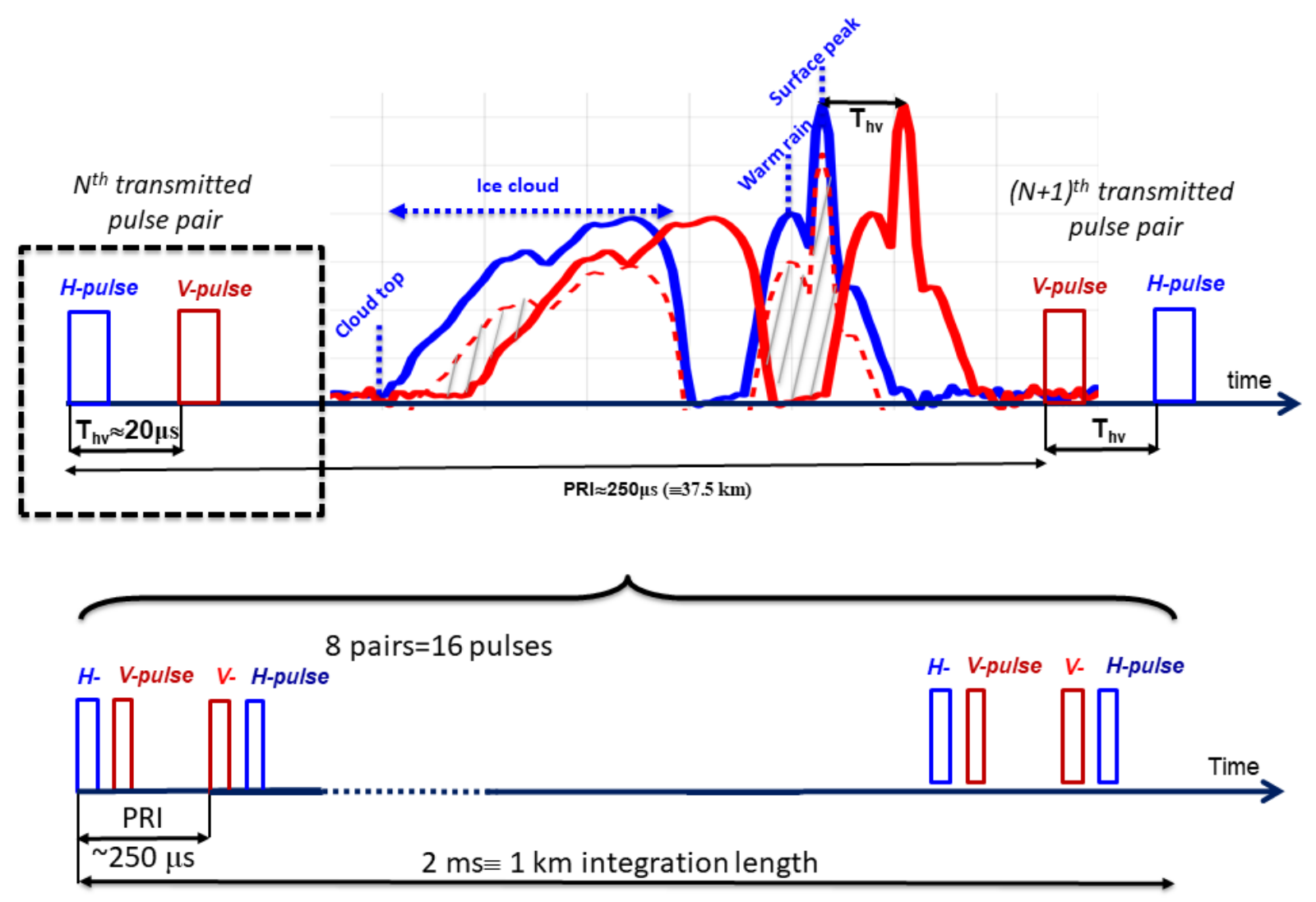

Figure 7. Bottom panel: the timing of the transmitted pulse sequence proposed for WIVERN with interlaced H-V pairs. A sequence of 8 pairs correspond to $2 \mathrm{~ms}$, equivalent to a $1 \mathrm{~km}$ distance along the scanning track. Note that the order of the polarization state of each pulse pairs is switched from pulse to pulse in order to cancel out differential phase shift effects between the two channels. Top panel: example of the return echoes from a scene including an ice cloud, a cloud free region and warm rain above a strongly reflecting surface. The returns in the $\mathrm{H}$ channel are plotted in blue, those in the V channel (lagging by $20 \mu \mathrm{s}$ ) in red. The dashed red line corresponds to the interference caused by the blue $\mathrm{H}$ pulse encountering a depolarising target. A very high depolarisation ratio of -10 $\mathrm{dB}$ has been used to exacerbate this effect that leads to "ghosts" in the red H channel. The hatched areas represent ranges where the "ghosts" exceed the co-polar signal; in this case the one from the ground and the warm rain is much more serious (and appears shifted upward by circa $3 \mathrm{~km}$ in correspondence to the cloud free region) than the one caused by the large $\mathrm{Z}$ gradients at the top of the cloud. A similar reasoning applies to the H-channel (not shown for clarity of purpose).

in the co-polar channel for the first $M$ pulses of the pair and:

$Z_{2}(r)=Z_{c o}(r)+Z_{c x}\left(r-c T_{h v} / 2\right)$ 
for the second $M$ pulses of the pairs. The radar Doppler velocities also have a component associated with the hydrometeor and one with the surface. The former is given by:

$v_{D}^{a t m}(r)=\frac{\iiint_{V} v_{L O S}^{a t m} Z_{c o} G^{2} d V}{\iiint_{V} Z_{c o} G^{2} d V}$

where $V$ is the backscattering volume (coloured region in Fig. 6), $v_{L O S}^{a t m}$ is projection of the the satellite velocity minus the hydrometeor velocity (the result of the wind speed and the hydrometeor fall-speed) along the line of sight (LOS) and $Z_{c o}$ is the measured co-polar reflectivity factor; note that the ghosts echoes will have random phase, so will not produce any bias in the wind but only a loss of precision (Pazmany et al., 1999; Battaglia et al., 2018; Wolde et al., 2019). NUBF effects (Battaglia and Kollias, 2015; Battaglia et al., 2018) Eq. (12) can be assessed by setting the satellite velocity equal to zero and looking at the deviation of Doppler velocities.

Similarly to Eq. (5) the Doppler associated with the surface will be equal to:

$v_{D}^{\text {surf }}(r)=\frac{\int_{\Sigma} \frac{v_{L O S}^{\text {surf }} \sigma_{0}\left(\theta_{a}, \phi_{a}\right) f_{a}^{2}\left(\theta_{a}\right) e^{-2 \int_{0}^{r} k_{e x t}(s) d s}}{r^{4}} d \Sigma}{\int_{\Sigma} \frac{\sigma_{0}\left(\theta_{a}, \phi_{a}\right) f_{a}^{2}\left(\theta_{a}\right) e^{-2 \int_{0}^{r} k_{e x t}(s) d s}}{r^{4}} d \Sigma}$

where $v_{L O S}^{\text {surf }}$ is projection of the the satellite velocity onto the line of sight; here we assume that the surface is still but any movement could be added if, for instance, ocean currents were available.

\subsection{Polarization diversity pulse pair processing}

A complete description of the simulation of $I$ and $Q$ time series for a system adopting polarization diversity is described in Battaglia and Kollias (2015). Here we adopt a simpler approach and use theoretical results to derive the noisiness of the reflectivity and velocity fields.

For reflectivities, since the Doppler spectral widths, $\sigma_{v}$, are expected to exceed $3 \mathrm{~m} / \mathrm{s}$ for all scanning directions, we can consider reflectivity measurements, separated by a pulse repetition interval $(P R I)$, as independent (for instance the correlation function for $3 \mathrm{~m} / \mathrm{s}$ and a time lag equal to $250 \mu \mathrm{s}$ is 0.0072 ). Therefore the number of independent samples practically is identical to the number of samples. For each single pulse we simulate the total power $P$ as a combination of noise, $N$ (equal to $-18 \mathrm{dBZ}$ ) and signal, $S$ [equal to the expressions given in (10-11)] by using the fact the probability distribution of power is a simple exponential with a standard deviation equal to the mean (Doviak and Zrnić, 2006), i.e.:

$P_{\text {single pulse }}=-\log (r)(N+S)$

with $r$ is a random number uniformly distributed between 0 and 1 . Note that, since we oversample in range every $100 \mathrm{~m}$, the application of Eq. (14) must be performed before the deconvolution in range (Eq. 4) because oversampled reflectivities and Doppler velocities are not independent. Power is averaged along track by simply averaging single pulses powers. Since the WIVERN footprint moves at about $500 \mathrm{~km} / \mathrm{s}, 8$ pulses must be averaged per km for each of the two channels (Fig. 7).

Doppler velocities estimated via pulse-pair processing also have intrinsic noise associated with the phase and thermal noise and to the cross-polarization interference. Uncertainties depend on the signal to noise ratio $(S N R)$, the radar Doppler spectral 
width and the number of averaged samples (Battaglia et al., 2013; Illingworth et al., 2018a). Following Pazmany et al. (1999), the estimate of the variance of the mean Doppler velocity for $M$ independent pulse pair samples can be written as:

$$
\begin{aligned}
\operatorname{var}_{\hat{v}_{D}} & =\frac{1}{M} \frac{v_{N y}^{2}}{2 \pi^{2} \beta^{2}}\left[\left(1+\frac{1}{S N R}\right)^{2}+\frac{1}{S G R_{1}}+\frac{1}{S G R_{2}}+\frac{1}{S G R_{1} S G R_{2}}+\frac{1}{S N R\left(S G R_{1}+S G R_{2}\right)}-\beta^{2}\right] \\
\beta & \equiv e^{-\frac{4 \pi^{2} \sigma_{v}^{2} T_{h v}^{2}}{\lambda^{2}}} ; \quad S N R=\frac{S}{N} ; \quad S G R_{1}=\frac{Z_{c o}(r)}{Z_{c x}\left(r-c T_{h v} / 2\right)} ; \quad S G R_{2}=\frac{Z_{c o}(r)}{Z_{c x}\left(r+c T_{h v} / 2\right)} .
\end{aligned}
$$

5 A Gaussian random noise with standard deviation corresponding to Eq. (15) is added to the velocities, which are then folded back into the Nyquist interval, $v_{N y}=\frac{\lambda}{4 T_{h v}}$.
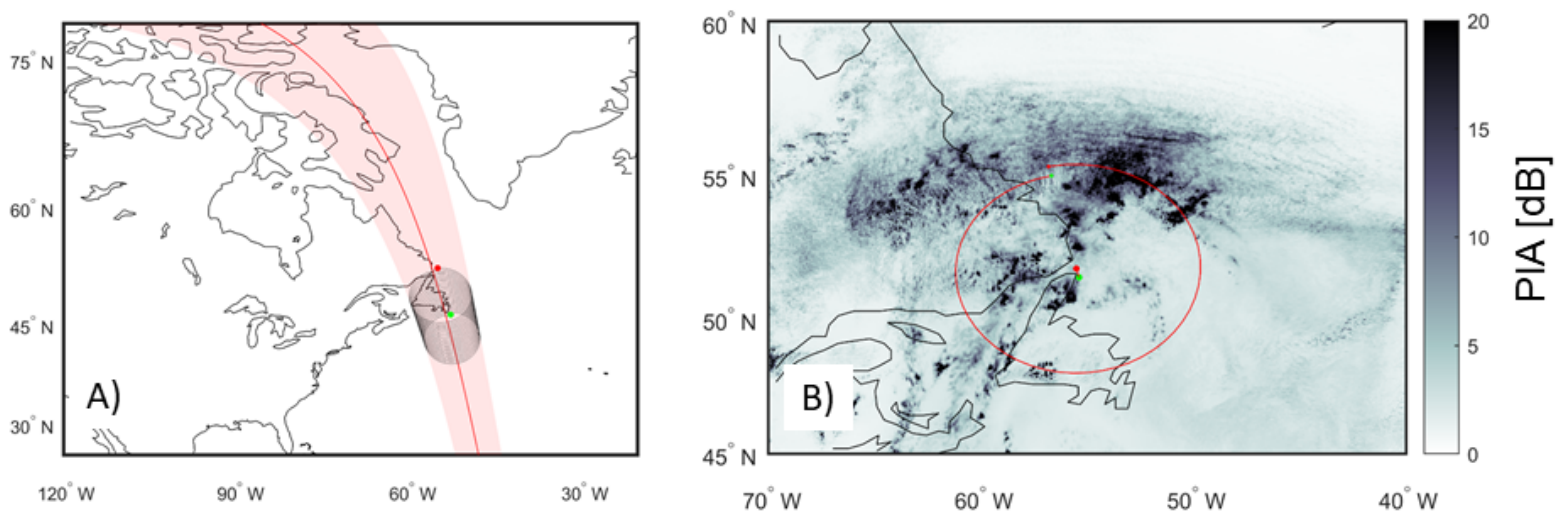

Figure 8. Panel A: WIVERN satellite track off the Labrador coast with the satellite ground track (red line), the scanning swath (shaded red region) and the radar footprints (black line) for 20 full revolutions of the conically scanning antenna corresponding to a flight time of $100 \mathrm{~s}$. Panel B: details of a single revolution of the WIVERN antenna. This rotation sample will be examined in detail later (see Figs. 11-14).

\subsection{Mispointing modelling}

For accurate winds the pointing of the radar beam formed by the antenna must be known very accurately; for instance a $140 \mu \mathrm{rad}$ uncertainty produces to a $1.0 \mathrm{~m} / \mathrm{s}$ LOS wind uncertainty. The antenna boresight direction can be identified by two angles: the elevation and the azimuthal angle (see Fig. 5); the former can be monitored by controlling the sea surface return range whereas the knowledge of the azimuthal angle is more challenging. The azimuth mispointing is usually described in terms of its frequency distribution by a Power Spectral Density (PSD). Previous industrial studies conducted for the SKIM mission Ardhuin et al. (2019) predict a PSD with a low frequency (orbit to seasonal scale) component dominated by the satellite stability and the antenna Thermo Elastic Distorsion (TED) and a high frequency component affected by antenna and satellite micro-vibrations. A schematic PSD for the azimuthal angle mispointing is sketched in Fig. 9. PSDs provide the input for our simulator. Time series representations of mispointing angles, $\Delta \phi$, can be produced by firstly constructing a frequency domain signal and then applying the Inverse Fast Fourier Transform (IFFT). The one-sided PSD in Fig. 9 is sampled at discrete 


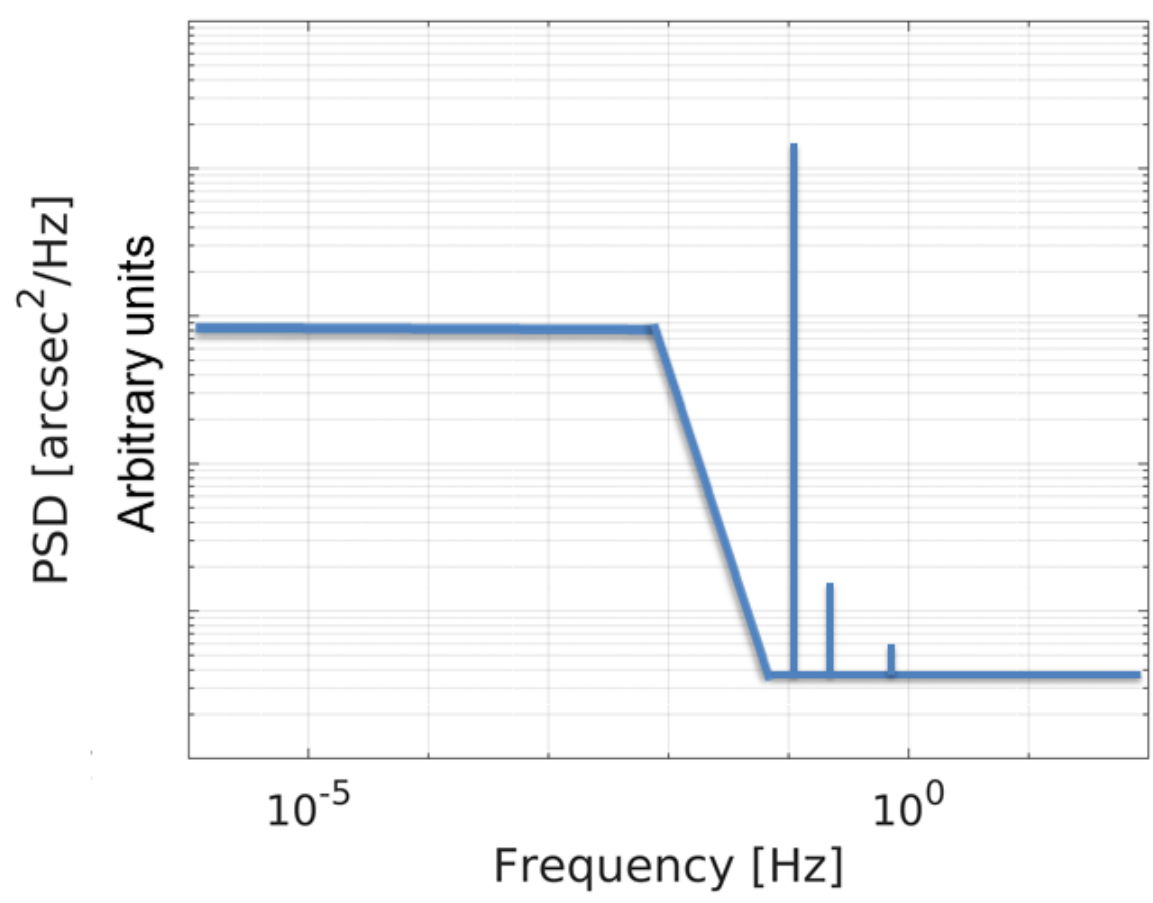

Figure 9. Conceptual model of the azimuth absolute knowledge error PSD. Contributions from different mechanisms are expected in different regions of the spectrum; for instance sharp peaks are expected in correspondence to the scan harmonics.

frequencies, going from zero to the Nyquist critical frequency $f_{c}$. The one-sided PSD is then mirrored into a two-sided power spectrum. Since the total power must be preserved, the values in the two-sided PSD are half the values of the one-sided PSD, except for the ones associated with the frequencies 0 and $\pm f_{c}$.

The amplitude of the two-sided spectrum of the signal is calculated from the two-sided PSD by taking the square root and 5 adding to each sample a random phase in the $[0,2 \pi]$ interval. The spectrum is forced to be conjugate symmetric, so that the IFFT returns a real-valued time series for the mispointing angle. An example of such a time series for a single antenna revolution is shown in Fig. 10 (left panel) with the corresponding LOS velocity error (right panel). The amplitude of the velocity error is a strong function of the azimuthal position. If $\phi$ is the azimuthal angle measured clockwise from the forward direction then the error can be approximated as:

$\Delta v_{m i s}=v_{\text {sat }} \sin \left(38^{\circ}\right)\left[\frac{1}{2} \cos (\phi) \Delta \phi_{m i s}^{2}+\sin (\phi) \Delta \phi_{\text {mis }}\right] \approx v_{\text {sat }} \sin \left(38^{\circ}\right) \sin (\phi) \Delta \phi_{\text {mis }}$,

which clearly shows that the error is minimised close to the forward and backward directions and amplified at side views. When inputting a realistic PSD as derived from initial industrial studies (internal communications, confidential) the error due to azimuthal mispointing remains always smaller than $0.17 \mathrm{~m} / \mathrm{s}$, thus it will provide a very small contribution to the Doppler velocity error budget. 

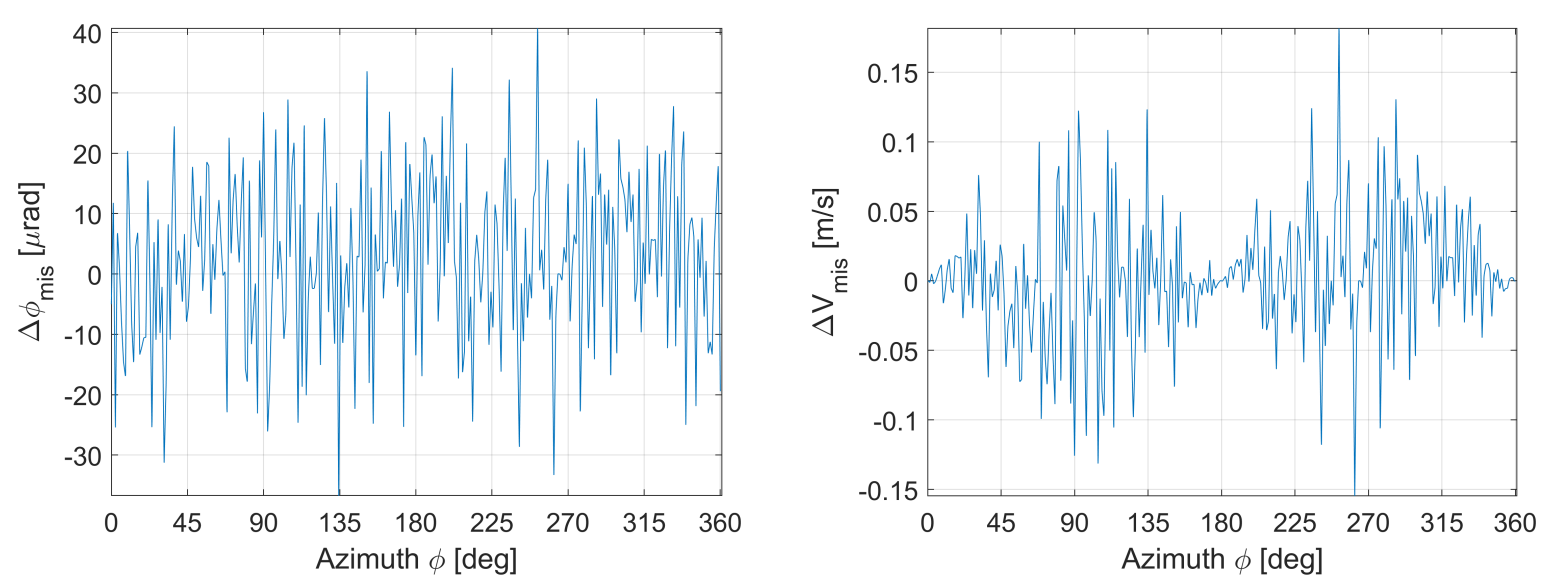

Figure 10. Left panel: One possible time series of the azimuthal scanning angle mispointing corresponding to a PSD like in Fig. 9 as provided by preliminary industrial studies. Right panel: LOS velocity error corresponding to the mispointing shown in the left panel. Mispointing errors corresponding to the PSD in Fig. 9 are generally smaller than $0.1 \mathrm{~m} / \mathrm{s}$, far lower than the precision of the Doppler observations.

\subsection{Radiometric mode}

WIVERN is also envisaged to have a radiometric mode. During the $250 \mu$ s time between transmitted pulse pairs, there will be a dedicated time (of the order of 10\%) with a dedicated broad bandwidth receiver for each receiver. The brightness temperatures in the two polarization modes are simulated by an Eddington radiative transfer model (Kummerow, 1993) by using the slant

5 one-dimensional approximation (Battaglia et al., 2005). Land emissivities are polarization independent and assumed to be equal 0.9 whereas ocean emissivities are computed via the TESSEM model (Prigent et al., 2017) with the $10 \mathrm{~m}$ wind and the SST from the model product.

\section{Applications of the E2E simulator}

\subsection{Case study: system over Labrador}

10 The simulator rationale is demonstrated for a case study simulating an overpass over Labrador. The satellite is moving northward and is scanning counterclockwise; the satellite ground track over North America is shown in Fig. 8 with a detail of the scanning pattern shown only for the region off the Labrador coast (Panel A). A full scan circle (5 s) is simulated in detail (Panel B): the slant view of the radar beam for the full revolution is used to compute the antenna weighted hydrometeor water content, 
$W C$, (Fig. 11A) and LOS winds (Fig. 11B):

$$
\begin{aligned}
W C_{A W}(r) & =\frac{\iiint_{V} W C G^{2} d V}{\iiint_{V} G^{2} d V}, \\
v_{A W}(r) & =\frac{\iiint_{V} v_{L O S} G^{2} d V}{\iiint_{V} G^{2} d V} .
\end{aligned}
$$

A variety of cloud and precipitation types is present in the scene with multiple layers of ice and liquid clouds at different 5 heights and with disparate thicknesses. The LOS winds show a characteristic alternating sign behaviour associated with the conically scanning geometry and present some strong vertical wind shears.
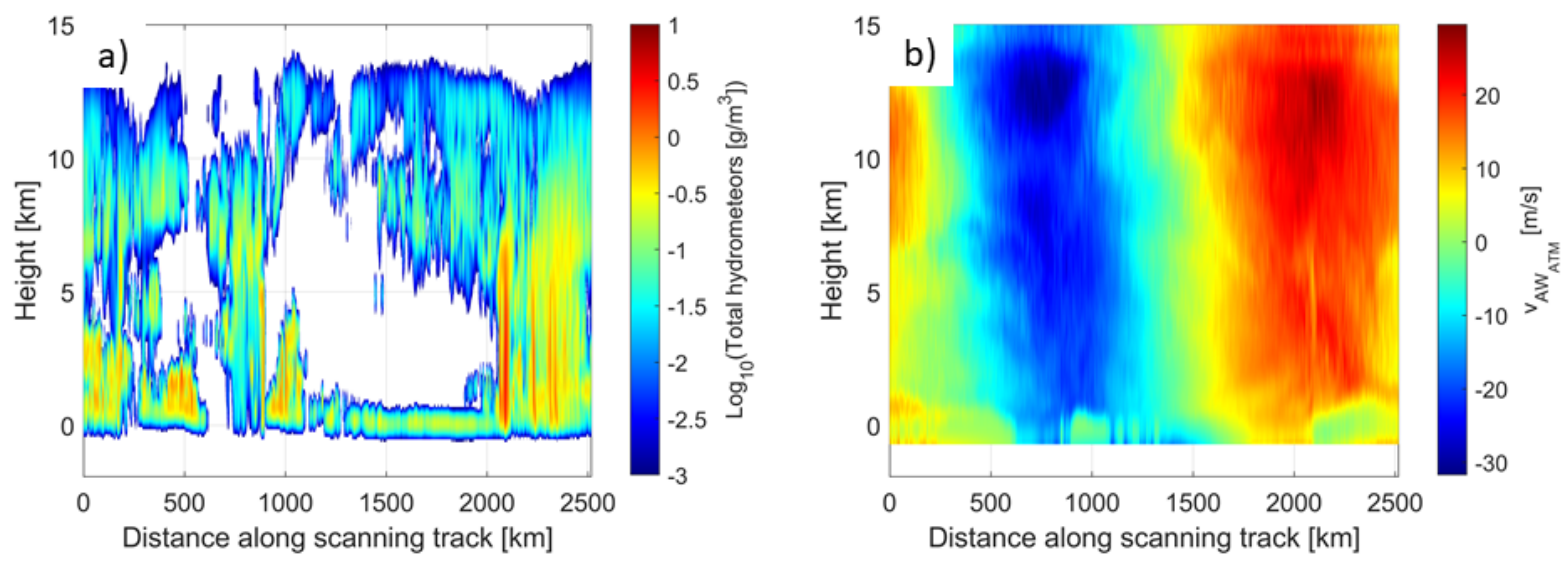

Figure 11. Antenna weighted hydrometeor content in $\mathrm{g} / \mathrm{m}^{3}$ expressed in base-10 logarithmic scale (Panel A) and LOS winds (Panel B) in correspondence to the revolution shown in Fig. 8B. Only hydrometeor contents above $1 \mathrm{mg} / \mathrm{m}^{3}$ are shown. The change in velocity reflects the rotation of the antenna around the weather system as shown in Fig. 2B.

Reflectivities and mean Doppler velocities for the atmospheric and surface targets computed according to the methodology described in Sect. 2.3 are shown in Fig. 12. The atmospheric reflectivity mirrors the hydrometeor contents but with a region of strong attenuation corresponding to heavy rain from $2000 \mathrm{~km}$ onward. Only reflectivities above $-30 \mathrm{dBZ}$ are plotted. The surface reflectivity and Doppler velocities are shown in the bottom panels. The reflectivity of the surface is clearly modulated by two effects: atmospheric attenuation (very strong e.g. at about 900 and $1100 \mathrm{~km}$ in the along-track coordinate) and $\sigma_{0}$ variability with large discontinuities at sea-land transitions (e.g. at about 670, 805 and $1390 \mathrm{~km}$ in the along-track coordinate). Note that the clutter signal tends to decrease to very low levels $(<-30 \mathrm{dBZ})$ at a height of $1 \mathrm{~km}$. This confirms previous findings (Illingworth et al., 2020); however, attention should be paid in future work to antenna sidelobes that can effectively enhance clutter contamination on Doppler signal especially over land (see Fig. 8 in Illingworth et al. (2020)).

The surface Doppler (sampled at very fine range resolution) shows its characteristic behaviour with zero velocity at the surface range and a pattern of positive and negative velocities at other ranges with a strong dependence on the scanning azimuthal angle, which is used as an alternative $\mathrm{x}$-axis coordinate in the bottom right panel. The azimuthal angle is measured 

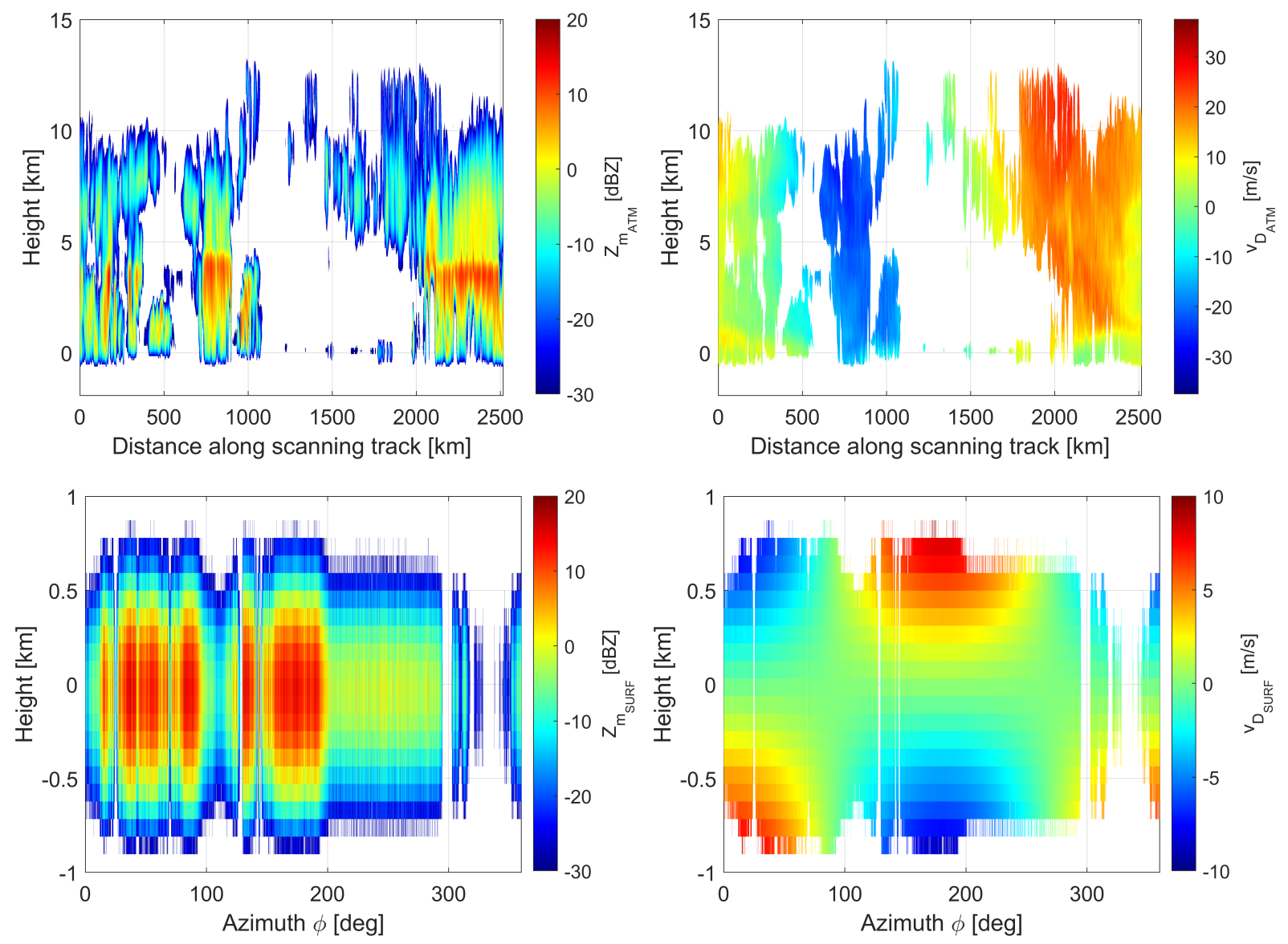

Figure 12. Atmospheric (top) and surface (bottom) reflectivities (left panels) and Doppler velocities (right panels) in correspondence to the revolution shown in Fig. 8B. We have used interchangeably the azimuth position angle $\phi$ (measured clockwise and equal to zero when the antenna is looking forward along the travelling direction) and the distance along the scanning track. The reflectivity (top left) clearly shows region of high attenuation below the freezing level (located at about $4.5 \mathrm{~km}$ ) especially in correspondence to distances along scanning track between 2200 and $2300 \mathrm{~km}$. In the lower panels the surface contributions are shown in the $\pm 1 \mathrm{~km}$ altitude region. Note that the ground clutter due to the $500 \mathrm{~m}$ long pulse is higher over the land than over the sea and in regions with no attenuation (bottom left panel).

clockwise from the forward looking direction (where it is in the same direction as the satellite motion). When the radar is side-looking the surface appears perfectly still at all altitudes whereas when the radar is looking in the forward or backward directions there is a strong variability with altitudes; as a result, the bias in Doppler velocities induced by clutter contamination will depend on the signal to clutter ratio, the altitude and the azimuthal scanning direction. Overall, when averaging over

5 heights and azimuthal angles, the clutter contamination will produce a bias towards zero Doppler velocities, i.e. the ground clutter will tend to mute the boundary layer winds. 

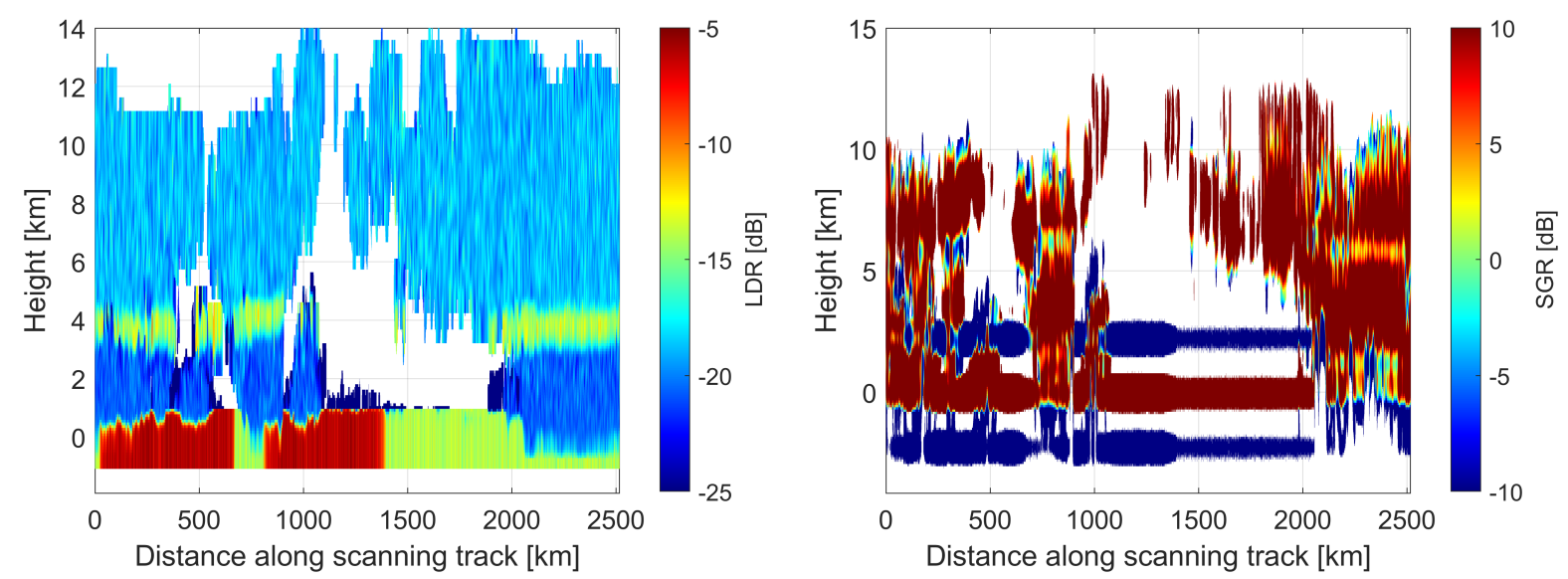

Figure 13. Linear depolarization ratio (LDR, left) and signal to ghost ratio (SGR, right) in correspondence to the revolution shown in Fig. 8B as a function of height. The LDR clearly shows the significant depolarizations by the melting layer that is straddling the heights around $4 \mathrm{~km}$ and by the surfaces with clear transitions from strong depolarizing land to weaker depolarizing sea surfaces. When no clouds are present the cross talk signal the SGR becomes $-\infty$; thus the SGR is capped at $-10 \mathrm{~dB}$. This is the case for several instances at an height of \pm 2.3 (which corresponds to a slant range of $3 \mathrm{~km}$ ) in coincidence with surface-cross talk.

The $L D R$ values shown in the left panel of Fig. 13 clearly have highest values in the melting layer and the land surfaces. These two regions are the major sources of ghosts as can be deduced by looking at the $S G R$ (right panel of Fig. 13), with strongly negative values associated with the ghosts generated by the surface at heights straddling $\pm 2.3 \mathrm{~km}$ and with larger $S G R \mathrm{~s}$ at about $6 \mathrm{~km}$ associated with the ghosts caused by the melting layer. Ghosts tend also to appear at cloud top, a phenomenon which, if not accounted for, will tend to artificially thicken high clouds.

The two panels of Fig. 14 show simulations of WIVERN products: reflectivities (left) and LOS Doppler velocities (right panel) after $1 \mathrm{~km}$ along-track integration. The reflectivities $\left(Z(r)=0.5\left[Z_{1}(r)+Z_{2}(r)\right]\right)$ are the averages of $M=8$ pairs and include signal and noise; at such an integration length the sensitivity (after noise subtraction) is expected to be $-22.5 \mathrm{dBZ}$, i.e. $5 \log _{10}(8)=4.5 \mathrm{~dB}$ better than the single-pulse sensitivity. Only regions with signal exceeding this level are plotted in Fig. 14 .

The presence of ghosts arising from surface cross-talk is obvious around an altitude of $\pm 2.3 \mathrm{~km}$; because of the considerably higher $\sigma_{0}^{H V}$ over land the minimum $S G R$ [computed as the minimum value between $S G R_{1}$ and $S G R_{2}$ as defined in Eq. (16), see the right panel of Fig. 13] the ghosts are significantly smaller over land than over ocean (where they almost disappear below the noise level). The ghosts only marginally affect the LOS velocities (Fig. 14, right panel); they only cause an increase in the standard deviation of the Doppler velocities according to Eq. (15) in the regions with detectable signal. Velocities are limited to the Nyquist interval $(-40,+40) \mathrm{m} / \mathrm{s}$ which is broad enough to capture the maximum amplitude of the LOS winds in this scene (Fig. 11B). In regions with very low $S N R$ or $S G R$ (e.g. around $-2.3 \mathrm{~km}$ below the surface) the estimated velocities practically 

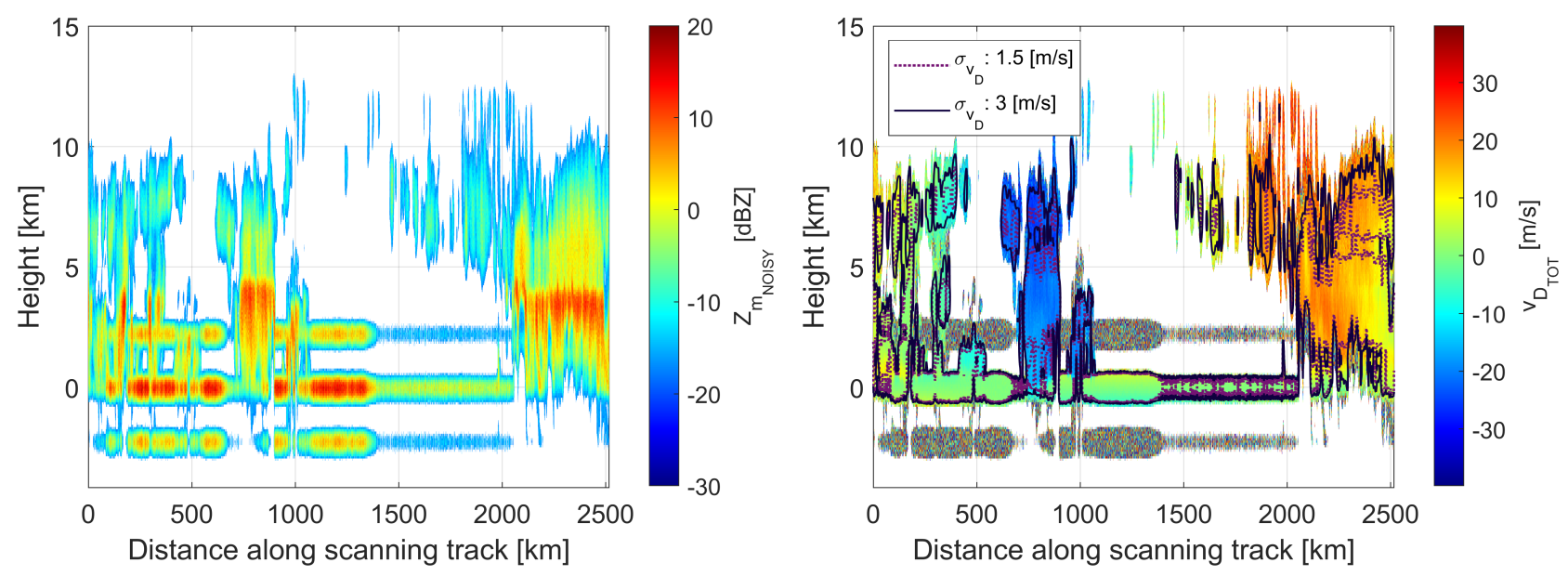

Figure 14. Reflectivities and Doppler results corresponding to a full revolution of the WIVERN antenna as shown in Fig. 12 for an integration length of $1.0 \mathrm{~km}(M=8)$. Left panel: simulations of the WIVERN reflectivities (signal+noise) with ghost echoes at $2.3 \mathrm{~km}$ above and below the ground due to the depolarisation by the surface leading to ghost echoes where there is no real cloud. Right panel: WIVERN retrieved line-of-sight Doppler velocities after performing the polarization diversity pulse pair processing. continuous (dashed) black contour lines correspond to Doppler velocity precision of $3(1.5) \mathrm{m} / \mathrm{s}$ according to Eq. (15)). Note that the ghost echoes have lower precision.

become random numbers within the Nyquist interval (hence the grainy texture in the graph). Otherwise the estimated LOS Doppler velocities well resemble the LOS winds depicted in Fig. 11B.

Another WIVERN product is the $\mathrm{H}$ and V-polarized brightness temperatures (Fig. 15). Due to the difference in emissivities there is a clear separation of the vertical and horizontal $T_{B} \mathrm{~s}$ over the ocean. With increasing optical thicknesses the two $T_{B} \mathrm{~s}$ tend to get closer and closer. This $T_{B}$ enhancement due to emission over cold backgrounds is expected to be useful for rain retrievals. In fact, because of the reduced and more variable ocean NRCS, surface reference technique-based PIA estimates will be more challenging and more sparse in WIVERN configuration than for nadir-looking radars; in addition $T_{B} \mathrm{~S}$ are known to have a better sensitivity than PIAs (Battaglia et al., 2020), i.e. they will produce a detectable signal at smaller optical thicknesses (compare blue and red line variability). The coincident sampling of reflectivity profiles and $T_{B} \mathrm{~s}$ will be unique and provide insights into supercooled liquid clouds in snow over the ice-free ocean (Battaglia and Panegrossi, 2020) and the evolution of large ice particles in deep convection.

\subsection{WIVERN performance assessment}

The E2E simulator represents a perfect tool to study the performances of the WIVERN mission. Apart from the errors related to the Doppler estimators in the pulse-pair processing [formula (15)] and the mispointing (Tanelli et al., 2005) there are other sources of uncertainties in polarization diversity Doppler radar measurements such as errors linked to wind shears 


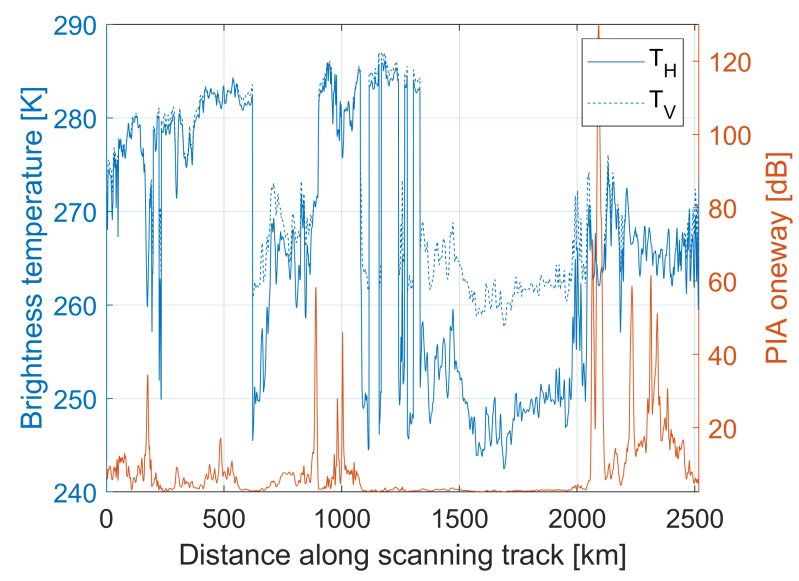

Figure 15. Simulated brightness temperatures for $V$ (continuous blue) and $H$ (dashed blue) polarization. The total one-way PIA (red line) is also given for reference. Results correspond to a full revolution of the WIVERN antenna as shown in Fig. 12.
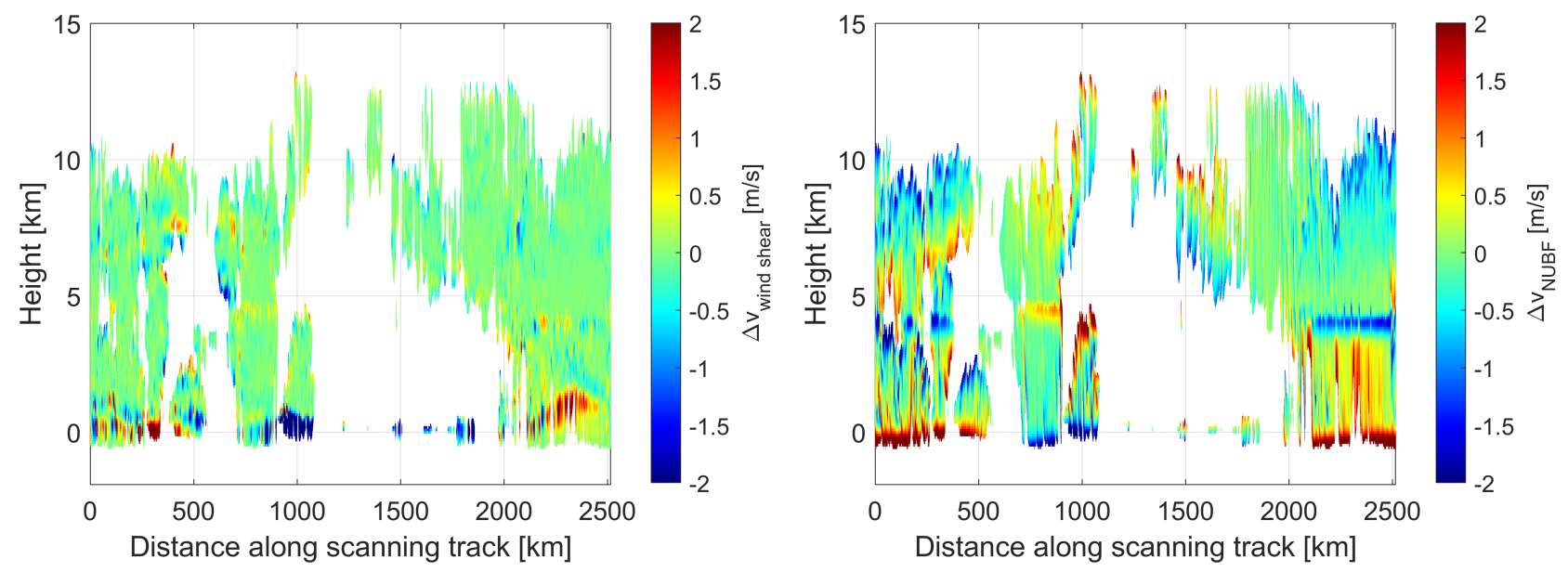

Figure 16. Errors induced by wind shear (left) and NUBF (right) in correspondence to the revolution shown in Fig. 8B.

either associated with the platform motion (Tanelli et al., 2002; Kollias et al., 2014) or to the atmospheric winds (Battaglia et al., 2018), to clutter contamination (Illingworth et al., 2020), to aliasing (Battaglia et al., 2013; Sy et al., 2014), to multiple scattering (Battaglia and Tanelli, 2011). The contribution of each of these errors can be quantified unambiguously by running two simulations where the effect is turned on and off. 


\subsubsection{Wind shear errors}

For instance the wind shear errors which tend to occur when reflectivity and velocity gradients are present at the same time within the backscattering volume, as can happen at the boundaries of clouds, can be computed from the difference between $v_{A W}$ in Eq. (18) and the expression of $v_{D}^{a t m}$ in Eq. (12) with $v_{\text {sat }}$ set to 0. Results are shown in the left panel of Fig. 16 5 in correspondence to the revolution shown in Fig. 8B. Strong wind shears appear in this case at near-surface altitudes (see Fig. 11b), which results in significant wind shear errors exceeding $\pm 1 \mathrm{~m} / \mathrm{s}$ affecting the measurements at the low altitudes, but Fig. 16 shows that these errors impact only limited regions and are close to zero for most areas within the observed scene.

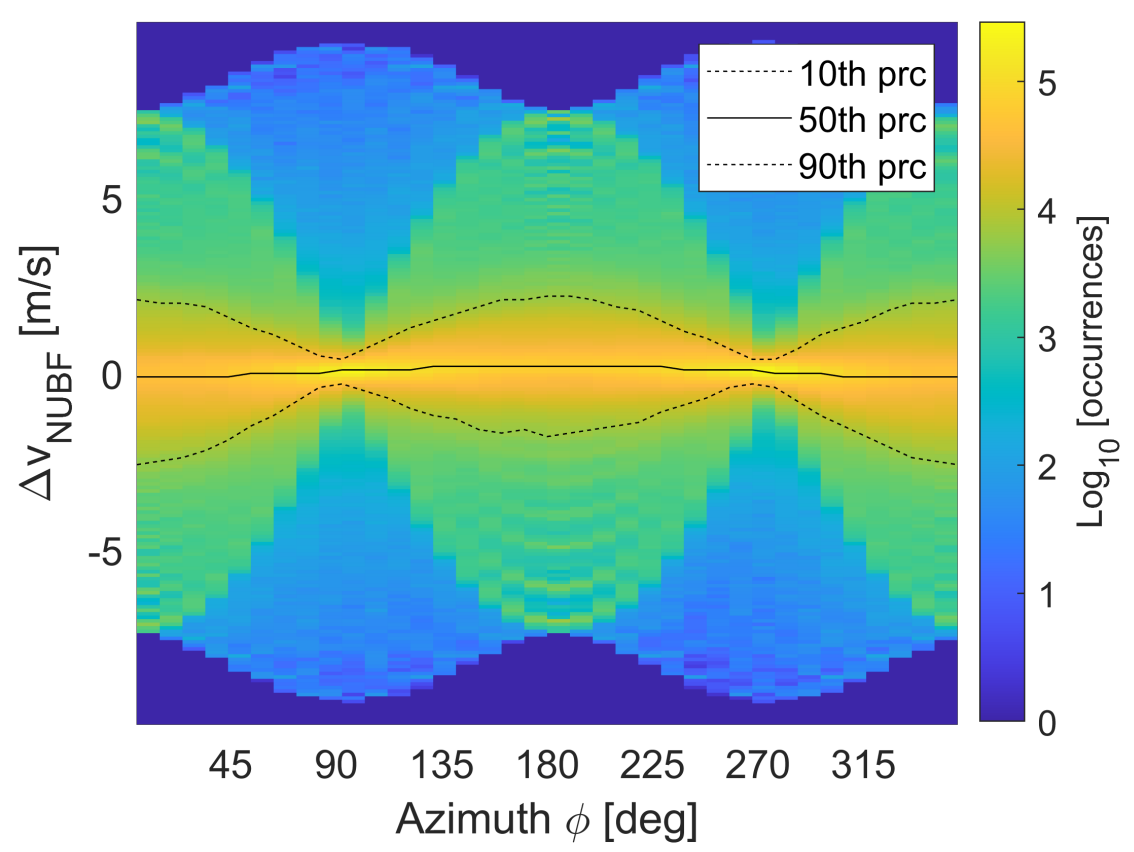

Figure 17. NUBF-induced error as a function of the azimuthal scanning angle. The color modulates the $\log _{10}$ of the number of occurrences. The statistics is computed for 20 orbits over the scene depicted in Figs. 11-14. The dotted lines represent the 10th and 90th percentile (typically with absolute value lower than $2.0 \mathrm{~m} / \mathrm{s}$ ) whereas the continuous line corresponds to the median value (always very close to zero because many NUBF errors are equal and opposite so will tend to cancel out).

\subsubsection{Non uniform beam filling: satellite motion-induced biases}

Similarly, estimates of the NUBF errors can be obtained by comparing the expression of $v_{D}^{a t m}$ in Eq. (12) with and without $v_{\text {sat }}$ set to 0 . 
Because of the different directions of the satellite velocity with respect to the antenna boresight when changing the scanning position, the NUBF errors depend on the azimuth scanning angle. The satellite velocity produces an apparent wind shear across the backscattering volume, with velocities ranging from -3.4 (-4.4) to $+3.4(4.4) \mathrm{m} / \mathrm{s}$ at forward/backward- (side-) looking configurations across the $3 \mathrm{~dB}$ WIVERN footprint. When coupled with a reflectivity gradient [computed along the direction orthogonal to the boresight and lying in the plane generated by the satellite velocity and by the antenna boresight (Battaglia et al., 2013)] this satellite-motion-induced velocity shear will produce a NUBF bias. The right panel of Fig. 16 shows NUBF errors for the revolution of Fig. $8 \mathrm{~B}$ and clearly demonstrates that the effect can be of several $\mathrm{m} / \mathrm{s}$, is strongly azimuth angle dependent (typically minimised at side view, e.g. for a distance along scanning track of about 625 and $1250 \mathrm{~km}$ ) and is driven by vertical gradients (e.g. strongly enhanced at the cloud top and in the melting layer). We have computed NUBF statistics for 20 full revolutions of the Labrador scene depicted in Fig. 8A: the distribution of the NUBF Doppler velocity biases as a function of the azimuthal scanning angle is shown in Fig. 17. In general the effect is maximum in the forward and backward directions $\left(\phi=0^{\circ}, 180^{\circ}\right)$ because in these directions the error is partly coupled with the vertical gradients of reflectivity; as a result, opposite biases are generally present in the upper troposphere and in the bright band (where $Z$ is sharply decreasing with height) and in the lower troposphere where, due to attenuation, reflectivities are increasing with height. At side view $\left(\phi=90^{\circ}, 270^{\circ}\right)$ the error is coupled only with the horizontal gradients of reflectivity, which may not be well captured by the model due to its coarse horizontal resolution; thus NUBF errors may be underestimated by our simulations at side view. Overall NUBF errors are within the requirements with the 10th and 90th percentiles exceeding $2 \mathrm{~m} / \mathrm{s}$ only at backward and forward viewing. Due to the conically scanning symmetry NUBF errors are equal and opposite when considering corresponding scanning directions in the forward and backward segment of the scan. When averaging winds over spatial scales of the order of $20 \mathrm{~km}$ or more (see Tab. 1) this will practically eliminate the NUBF bias and only worsen the precision of the LOS winds.

\section{Conclusions and future work}

This study introduces a state-of-the-art end-to-end simulator tailored to simulating space-borne conically scanning Doppler radars adopting polarization diversity with the inclusion of a radiometric mode. The "WIVERN" configuration as proposed to the ESA-Earth Explorer 11 call (see specifics in Tab. 3) has been implemented in this study. The simulator reproduces the satellite orbit, the radar scanning geometry and the illumination of an atmospheric scene extracted from a global atmospheric circulation model providing fine resolution vertical profiles of winds and clouds. The coupling between the orbit and the atmospheric model allows global scale simulations of mission observables, i.e. reflectivities and Doppler velocities of atmospheric targets. In addition, surface modelling accounts for the clutter returns from land and sea surfaces. The simulator also outputs estimates of Doppler measurement errors, such as those due to intrinsic noise, to cross-talk noise between the two diversely polarized channels and introduced in presence of reflectivity gradients (wind shear and non-uniform beam filling errors). Additional disturbances originate from the antenna azimuthal mispointing errors, represented in terms of an absolute knowledge error power spectrum. 
Preliminary findings show that mispointing errors associated with the antenna azimuthal mispointing are expected to be lower than $0.3 \mathrm{~m} / \mathrm{s}$ (and strongly dependent on the antenna azimuthal scanning angle), wind shear and non-uniform beam filling errors have generally negligible biases when full antenna revolutions are considered, NUBF causes random errors strongly dependent on the antenna azimuthal scanning angle but typically lower than $1 \mathrm{~m} / \mathrm{s}$ and cross-talk effects are well predictable so that areas affected by strong cross-talk noise can be flagged. The noise random errors are dependent on the SNR and the possible presence of ghosts and can be reduced by averaging over a higher number of pulses (i.e. by using a longer integration time). In summary our results show that the quality of the Doppler appears to strongly depend on several factors: the strength of the cloud reflectivity, the antenna pointing direction relative to the satellite motion, the presence of strong reflectivity and/or wind gradients, the strength of the surface clutter. Overall, the total wind errors seem to meet the mission requirements in a good portion of the clouds detected by the WIVERN radar, which is a very encouraging finding at the beginning of Phase 0 studies.

The characterization of the errors and the isolation of each single error source makes the E2E simulator a perfect tool to verify mission performances and compliance with requirements, which will be part of the Phase 0 studies beginning in December 2021. Different problematic areas will be investigated (with, possibly, the introduction of new features, see Tab. 2).

1. By changing the antenna gain [Eq. 1)] it will be possible to study the impact of antenna side-lobes in affecting the minimum height close to the surface at which winds can be observed by the WIVERN radar without suffering significant biases from the clutter return.

2. By modifying the point target response it will be possible to define the optimal trade-off between sensitivity and effective vertical resolution; this could also include studies related to pulse compression and effects associated with the range sidelobes.

3. Different modes could be employed for WIVERN operations including an interlaced LDR mode and different $T_{h v}$ interleaved modes. Such modes, very beneficial for identifying the ghost returns and for optimizing noise measurement and aliasing errors, could be optimized via E2E simulations.

4. More sophisticated surface modelling could be introduced by including the dependence on the surface winds over ocean and different surface types/orography over land.

5. Cloud scenes at finer horizontal resolution $(\lesssim 1 \mathrm{~km})$ that resolve convection could be used in the simulator at regional (if not global) scale; this will enable to evaluate WIVERN performances in presence of convective motions.

6. A multiple scattering module based on the two-stream approximation (Hogan and Battaglia, 2008) could be applied to the 1D WIVERN slant column and used to flag multiple scattering contaminated profiles.

7. Additional polarimetric variables like specific differential phase $\left(K_{D P}\right)$ and the co-polar cross-correlation coefficient $\left(\rho_{H V}\right)$ could be simulated. 
8. Further studies on mispointing effects will be performed once power spectral densities of azimuth and elevation knowledge error will be better specified by industrial studies. In particular the E2E simulator will be able to assess how frequently and with which accuracy the surface return could be used to check the elevation pointing.

9. The E2E simulator will also serve as the basis to develop mitigation algorithms for NUBF, wind shear, mispointing and vertical wind corrections that will be needed in order to produce horizontal line of sight winds, which will be the product directly assimilated by numerical weather prediction models.

Thanks to its modular structure the simulator can be easily adapted to different orbits, a gamut of scanning geometries (e.g. cross track) and various frequencies (by simply changing the look-up-tables). Therefore the simulator could be applied to simulate other space-borne Doppler atmospheric radars as well. formance Study, ESA Contract no. 4000130864/20/NL/CT. This research used the Mafalda cluster at Politecnico di Torino. 


\section{References}

Ardhuin, F., Brandt, P., Gaultier, L., Donlon, C., Battaglia, A., Boy, F., Casal, T., Chapron, B., Collard, F., Cravatte, S., Delouis, J.-M., DeWitte, E., Dibarboure, G., Engen, G., Johnsen, H., Lique, C., PacoLopez-Dekker, Maes, C., Martin, A., Mari, L., Menemenlis, D., Nouguier, F., Peureux, C., Rampal, P., Ressler, G., Rio, M.-H., Rommen, B., Shutler, J. D., Suess, M., Tsamados, M., Ubelmann, C., van Sebille, E., van der Vorst, M., and Stammer, D.: SKIM, a candidate satellite mission exploring global ocean currents and waves, Frontiers, 2019.

Battaglia, A. and Kollias, P.: Error Analysis of a Conceptual Cloud Doppler Stereoradar with Polarization Diversity for Better Understanding Space Applications, J. Atmos. Ocean Technol., 32, 1298-1319, https://doi.org/10.1175/JTECH-D-14-00015.1, https://doi.org/10.1175/ JTECH-D-14-00015.1, 2015.

Battaglia, A. and Panegrossi, G.: What Can We Learn from the CloudSat Radiometric Mode Observations of Snowfall over the Ice-Free Ocean?, Radio Sci., 12, https://doi.org/10.3390/rs12203285, https://www.mdpi.com/2072-4292/12/20/3285, 2020.

Battaglia, A. and Tanelli, S.: DOMUS: DOppler MUltiple-Scattering Simulator, IEEE Trans. Geosci. Remote Sens., 49, 442-450, https://doi.org/10.1109/TGRS.2010.2052818, 2011.

Battaglia, A., Prodi, F., Porcu, F., and Shin, D.-B.: Measuring Precipitation from space: EURAINSAT and the future, chap. 3D effects in MW radiative transport inside precipitating clouds: modeling and applications, V. Levizzani and P. Bauer and F. J. Turk, Kluwer Academic edn., 2005.

Battaglia, A., Tanelli, S., and Kollias, P.: Polarization Diversity for Millimeter Spaceborne Doppler Radars: An Answer for Observing Deep Convection?, J. Atmos. Ocean Technol., 30, 2768-2787, https://doi.org/10.1175/JTECH-D-13-00085.1, https://doi.org/10.1175/ JTECH-D-13-00085.1, 2013.

Battaglia, A., Wolde, M., D’Adderio, L. P., Nguyen, C., Fois, F., Illingworth, A., and Midthassel, R.: Characterization of Surface Radar Cross Sections at W-Band at Moderate Incidence Angles, IEEE Trans. Geosci. Remote Sens., 55, 3846-3859, 10.1109/TGRS.2017.2682423, 2017.

Battaglia, A., Dhillon, R., and Illingworth, A.: Doppler W-band polarization diversity space-borne radar simulator for wind studies, Atm. Meas. Tech., 11, 5965-5979, https://doi.org/10.5194/amt-11-5965-2018, https://www.atmos-meas-tech.net/11/5965/2018/, 2018.

Battaglia, A., Kollias, P., Dhillon, R., Lamer, K., Khairoutdinov, M., and Watters, D.: Mind-the-gap Part II: Improving quantitative estimates of cloud and rain water path in oceanic warm rain using spaceborne radars, Atm. Meas. Tech. Disc., 2020, 1-29, https://doi.org/10.5194/amt-2020-80, https://www.atmos-meas-tech-discuss.net/amt-2020-80/, 2020.

Bauer, P., Thorpe, A., and Brunet, G.: The quiet revolution of numerical weather prediction, Nature, 525, 47-55, doi:10.1038/nature14956, 2015.

30 Bohren, C. F. and Huffman, D. R.: Absorption and Scattering of Light by Small Particles, John Wiley \& Sons, New York, 1983.

Bringi, V. N. and Chandrasekar, V.: Polarimetric Doppler Weather Radar, Principles and applications, Cambridge University Press, pp 266, 2001.

Dellaripa, E. M. R., Funk, A., Schumacher, C., Bai, H., and Spangehl, T.: Adapting the COSP Radar Simulator to Compare GCM Output and GPM Precipitation Radar Observations, J. Atmos. Ocean Technol., 38, 1457 - 1475, https://doi.org/10.1175/JTECH-D-20-0089.1, https://journals.ametsoc.org/view/journals/atot/38/9/JTECH-D-20-0089.1.xml, 2021.

Doviak, R. J. and Zrnić, D. S.: Doppler Radar and Weather Observations, Dover, Mineiola, NY, second edition, 2006. 
Haynes, J. M., Marchand, R. T., Luo, Z., Bodas-Salcedo, A., and Stephens, G. L.: A Multipurpose Radar Simulation Package: QuickBeam, Bull. Amer. Met. Soc., 88, 1723-1727, dOI: 10.1175/BAMS-88-11-1723, 2007.

Hogan, R. J. and Battaglia, A.: Fast Lidar and Radar Multiple-Scattering Models. Part II: Wide-Angle Scattering Using the TimeDependent Two-Stream Approximation, J. Atmos. Sci., 65, 3636-3651, https://doi.org/10.1175/2008JAS2643.1, https://doi.org/10.1175/ 2008JAS2643.1, 2008.

Horanyi, A., Cardinali, C., Rennie, M., and Isaksen, L.: The assimilation of horizontal line-of-sight wind information into the ECMWF data assimilation and forecasting system. Part I: The assessment of wind impact, Quart. J. Roy. Meteor. Soc., 141, 1223-1232, http:// dx.doi.org/10.1002/qj.2430, 2014.

Illingworth, A., Battaglia, A., and Delanoe, J.: WIVERN: An ESA Earth Explorer Concept to Map Global in-Cloud Winds, Precipitation and

Cloud Properties, in: 2020 IEEE Radar Conference (RadarConf20), pp. 1-6, https://doi.org/10.1109/RadarConf2043947.2020.9266286, 2020.

Illingworth, A. J., Battaglia, A., Bradford, J., Forsythe, M., Joe, P., Kollias, P., Lean, K., Lori, M., Mahfouf, J.-F., Melo, S., Midthassel, R., Munro, Y., Nicol, J., Potthast, R., Rennie, M., Stein, T. H. M., Tanelli, S., Tridon, F., Walden, C. J., and Wolde, M.: WIVERN: A New Satellite Concept to Provide Global In-Cloud Winds, Precipitation, and Cloud Properties, Bull. Amer. Met. Soc., 99, 1669-1687, https://doi.org/10.1175/BAMS-D-16-0047.1, https://doi.org/10.1175/BAMS-D-16-0047.1, 2018a.

Illingworth, A. J., Battaglia, A., Delanoe, J., Forsythe, M., Joe, P., Kollias, P., Mahfouf, J.-F., Potthast, R., Rennie, M., Tanelli, S., Viltard, N., Walden, C., Witschas, B., and Wolde, M.: WIVERN (WInd VElocity Radar Nephoscope), Proposal submitted to ESA in response to the Call for Earth Explorer-10 Mission Ideas, 2018b.

Kendon, E. J., Ban, N., Roberts, N. M., Fowler, H. J., Roberts, M. J., Chan, S. C., Evans, J. P., Fosser, G., and Wilkinson, J. M.: Do Convection-Permitting Regional Climate Models Improve Projections of Future Precipitation Change?, Bull. Amer. Met. Soc., 98 , 79 93, https://doi.org/10.1175/BAMS-D-15-0004.1, https://journals.ametsoc.org/view/journals/bams/98/1/bams-d-15-0004.1.xml, 2017.

Khairoutdinov, M. F. and Randall, D. A.: Cloud Resolving Modeling of the ARM Summer 1997 IOP: Model Formulation, Results, Uncertainties, and Sensitivities, J. Atmos. Sci., 60, 607-625, https://doi.org/10.1175/1520-0469(2003)060<0607:CRMOTA>2.0.CO;2, https://doi.org/10.1175/1520-0469(2003)060<0607:CRMOTA>2.0.CO;2, 2003.

Kollias, P., Tanelli, S., Battaglia, A., and Tatarevic, A.: Evaluation of EarthCARE Cloud Profiling Radar Doppler Velocity Measurements in Particle Sedimentation Regimes, J. Atmos. Ocean Technol., 31, 366-386, https://doi.org/10.1175/JTECH-D-11-00202.1, https://doi.org/ 10.1175/JTECH-D-11-00202.1, 2014.

Kummerow, C.: On the accuracy of the Eddington approximation for radiative transfer in the microwave frequencies, J. Geophys. Res., 98 , 2757-2765, 1993.

30 Matsui, T., Iguchi, T., Li, X., Han, M., Tao, W.-K., Petersen, W., L'Ecuyer, T., Meneghini, R., Olson, W., Kummerow, C. D., Hou, A. Y., Schwaller, M. R., Stocker, E. F., and Kwiatkowski, J.: GPM Satellite Simulator over Ground Validation Sites, Bull. Amer. Met. Soc., 94, 1653 - 1660, https://doi.org/10.1175/BAMS-D-12-00160.1, https://journals.ametsoc.org/view/journals/bams/94/11/bams-d-12-00160.1. $\mathrm{xml}, 2013$.

McNally, A. P.: A note on the occurrence of cloud in meteorologically sensitive areas and the implications for advanced infrared sounders,

Quart. J. Roy. Meteor. Soc., 128, 2551-2556, 2002.

Meneghini, R. and Kozu, T.: Spaceborne weather radar, Artech House, 1990. 
Mróz, K., Battaglia, A., Kneifel, S., von Terzi, L., Karrer, M., and Ori, D.: Linking rain into ice microphysics across the melting layer in stratiform rain: a closure study, Atm. Meas. Tech., 14, 511-529, https://doi.org/10.5194/amt-14-511-2021, https://amt.copernicus.org/ articles/14/511/2021/, 2021.

Pazmany, A. L., Galloway, J. C., Mead, J. B., Popstefanija, I., McIntosh, R. E., and Bluestein, H. W.: Polarization Diversity Pulse-Pair Technique for Millimeter-WaveDoppler Radar Measurements of Severe Storm Features, J. Atmos. Ocean Technol., 16, 1900-1911, https://doi.org/10.1175/1520-0426(1999)016<1900:PDPPTF>2.0.CO;2, https://doi.org/10.1175/1520-0426(1999)016<1900:PDPPTF>2. $0 . \mathrm{CO} ; 2,1999$.

Prigent, C., Aires, F., Wang, D., Fox, S., and Harlow, C.: Sea-surface emissivity parametrization from microwaves to millimetre waves, Quart. J. Roy. Meteor. Soc., 143, 596-605, https://doi.org/10.1002/qj.2953, https://rmets.onlinelibrary.wiley.com/doi/abs/10.1002/qj.2953, 2017.

Rennie, M. P., Isaksen, L., Weiler, F., de Kloe, J., Kanitz, T., and Reitebuch, O.: The impact of Aeolus wind retrievals on ECMWF global weather forecasts, Quart. J. Roy. Meteor. Soc., n/a, n/a, https://doi.org/https://doi.org/10.1002/qj.4142, https://rmets.onlinelibrary.wiley. com/doi/abs/10.1002/qj.4142, 2021.

Satoh, M., Stevens, B., Judt, F., Khairoutdinov, M., Lin, S. J., Putman, W. M., and Duben, P.: Global cloud-resolving models, Current Climate Change Reports, 5, 172-184, https://doi.org/10.1007/s40641-019-00131-0, https://doi.org/10.1007/s40641-019-00131-0, 2019.

Stevens, B., Satoh, M., Auger, L., Biercamp, J., Bretherton, C. S., Chen, X., Düben, P., Judt, F., Khairoutdinov, M., Klocke, D., Kodama, C., Kornblueh, L., Lin, S.-J., Neumann, P., Putman, W. M., Röber, N., Shibuya, R., Vanniere, B., Vidale, P. L., Wedi, N., and Zhou, L.: DYAMOND: the DYnamics of the Atmospheric general circulation Modeled On Non-hydrostatic Domains, Prog Earth Planet Sci, 6, https://doi.org/10.1186/s40645-019-0304-z, 2019.

Sy, O. O., Tanelli, S., Takahashi, N., Ohno, Y., Horie, H., and Kollias, P.: Simulation of EarthCARE Spaceborne Doppler Radar Products Using Ground-Based and Airborne Data: Effects of Aliasing and Nonuniform Beam-Filling, IEEE Trans. Geosci. Remote Sens., 52, 1463-1479, https://doi.org/10.1109/TGRS.2013.2251639, 2014.

Tanelli, S., Im, E., Durden, S. L., Facheris, L., and Giuli, D.: The Effects of Nonuniform Beam Filling on Vertical Rainfall Velocity Measurements with a Spaceborne Doppler Radar, J. Atmos. Ocean Technol., 19, 1019-1034, https://doi.org/10.1175/15200426(2002)019<1019:TEONBF>2.0.CO;2, https://doi.org/10.1175/1520-0426(2002)019<1019:TEONBF>2.0.CO;2, 2002.

Tanelli, S., Im, E., Mascelloni, S. R., and Facheris, L.: Spaceborne Doppler radar measurements of rainfall: correction of errors induced by pointing uncertainties, J. Atmos. Ocean Technol., 22, 1676-1690, doi: 10.1175/JTECH1797.1, 2005.

Weiler, F., Rennie, M., Kanitz, T., Isaksen, L., Checa, E., de Kloe, J., Okunde, N., and Reitebuch, O.: Correction of wind bias for the lidar on-board Aeolus using telescope temperatures, Atm. Meas. Tech. Disc., 2021, 1-33, https://doi.org/10.5194/amt-2021-171, https: //amt.copernicus.org/preprints/amt-2021-171/, 2021.

Wolde, M., Battaglia, A., Nguyen, C., Pazmany, A. L., and Illingworth, A.: Implementation of polarization diversity pulse-pair technique using airborne W-band radar, Atm. Meas. Tech., 12, 253-269, https://doi.org/10.5194/amt-12-253-2019, https://www.atmos-meas-tech. net/12/253/2019/, 2019.

Data availability. The simulation inputs are available on request. The E2E simulator code is not yet available because part of on-going ESA studies. 
https://doi.org/10.5194/amt-2021-342

Preprint. Discussion started: 24 November 2021

(C) Author(s) 2021. CC BY 4.0 License.

(c) (1)

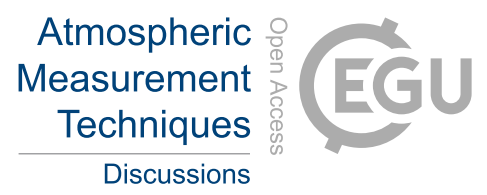

Author contributions. AB wrote most of the text and has built most of the modules of the simulator. PM has implemented the code in matlab and produced most of the figures. EC and LP have provided inputs for the orbital model and to the mispointing model plus they contributed to the scientific discussion. FS has provided supervision to PM and participated to the discussion on radar mispointing issues. PK and AI contributed to the discussion, the editing and the formulation of the WIVERN idea and definition.

5 Competing interests. The authors declare that they have no conflict of interest. 